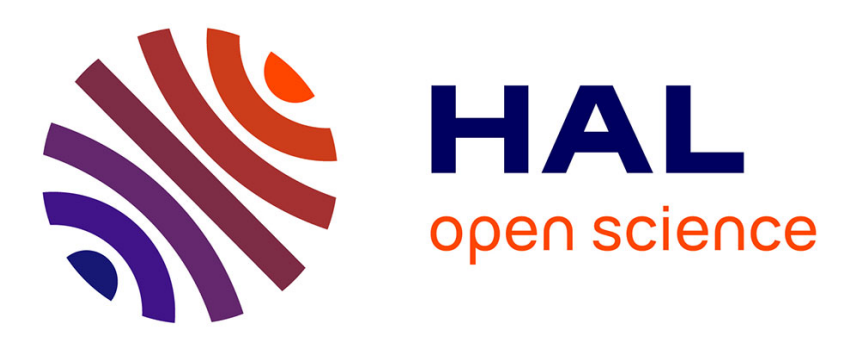

\title{
Effects of porosity and inertia on the apparent permeability tensor in fibrous media
}

Nicola Luminari, Christophe Airiau, Alessandro Bottaro

\section{To cite this version:}

Nicola Luminari, Christophe Airiau, Alessandro Bottaro. Effects of porosity and inertia on the apparent permeability tensor in fibrous media. International Journal of Multiphase Flow, 2018, vol.106, pp. 60-74. 10.1016/j.ijmultiphaseflow.2018.04.013 . hal-01811015

\section{HAL Id: hal-01811015 https://hal.science/hal-01811015}

Submitted on 8 Jun 2018

HAL is a multi-disciplinary open access archive for the deposit and dissemination of scientific research documents, whether they are published or not. The documents may come from teaching and research institutions in France or abroad, or from public or private research centers.
L'archive ouverte pluridisciplinaire HAL, est destinée au dépôt et à la diffusion de documents scientifiques de niveau recherche, publiés ou non, émanant des établissements d'enseignement et de recherche français ou étrangers, des laboratoires publics ou privés. 


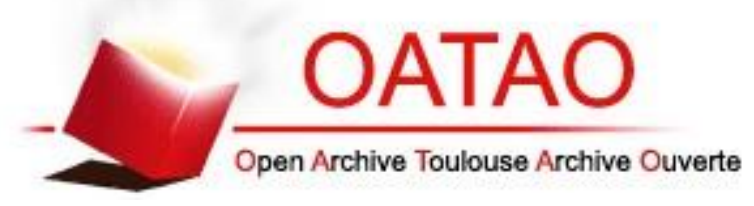

\section{Open Archive TOULOUSE Archive Ouverte (OATAO)}

OATAO is an open access repository that collects the work of Toulouse researchers and makes it freely available over the web where possible.

This is an author-deposited version published in : http://oatao.univ-toulouse.fr/ Eprints ID : 20150

To link to this article: DOI: 10.1016/j.ijmultiphaseflow.2018.04.013 URL : http://doi.org/10.1016/j.ijmultiphaseflow.2018.04.013

To cite this version : Luminari, Nicola $\rightleftharpoons$ and Airiau, Christophe Bottaro, Alessandro Effects of porosity and inertia on the apparent permeability tensor in fibrous media. (2018) International Journal of Multiphase Flow, vol.106. pp. 60-74. ISSN 0301-9322

Any correspondence concerning this service should be sent to the repository administrator: staff-oatao@ listes-diff.inp-toulouse.fr 


\title{
Effects of porosity and inertia on the apparent permeability tensor in fibrous media
}

\author{
Nicola Luminari ${ }^{a}$, Christophe Airiau ${ }^{\mathrm{a}}$, Alessandro Bottaro ${ }^{\mathrm{a}, \mathrm{b}, *}$ \\ a Institut de Mécanique des Fluides de Toulouse (IMFT), Université de Toulouse, CNRS, Toulouse, France \\ ${ }^{\mathrm{b}}$ DICCA, Università di Genova, 1 via Montallegro, Genova 16145, Italy
}

\begin{abstract}
A B S T R A C T
The flow in three-dimensional fibrous porous media is studied in the inertial regime by first simulating for the motion in unit, periodic cells, and then solving successive closure problems leading - after applying an intrinsic averaging procedure - to the components of the apparent permeability tensor. The parameters varied include the orientation of the driving pressure gradient, its magnitude (which permits to define a microscopic Reynolds number), and the porosity of the medium. All cases tested refer to situations for which the microscopic flow is steady. When the driving force is oriented in a direction which lies on the plane perpendicular to the fibers' axis, the results found agree with those available the literature. The fact that the medium is composed by bundles of parallel fibers favours a deviation of the mean flow towards the fibers' axis when the driving pressure gradient has even a small component along it, and this is enhanced by a decreasing porosity; this phenomenon is well quantified by the knowledge of the components of the permeability. Contrary to our initial expectations, for the over one hundred cases which we have simulated, the apparent permeability tensor remains, to a very good approximation, diagonal, a fact mainly related to the transversely isotropic nature of the medium. To obtain a complete, albeit approximate, database of the diagonal components of the apparent permeability tensor we have developed a metamodel, based on kriging interpolation, and carefully calibrated it. The resulting response surfaces can be invaluable in determining the force caused by the presence of inclusions in macroscopic simulations of the flow through bundles of fibers whose orientations and dimensions can vary in space and/or time.
\end{abstract}

\section{Introduction}

The flow through porous media is a problem of importance for several natural and technological applications. Since the original formulation by Darcy (1856), which relates the flow rate through a porous bed to the pressure drop across the bed's sides, many corrections have been made to account, for example, for viscous effects (Brinkman, 1949) or for the consequences of inertia (Forchheimer, 1901). All of the cited works are of empirical nature, but a volume averaging approach has been able to recover all of these formulations rigorously starting from the Navier-Stokes equations (Whitaker, 2013).

The theory requires the knowledge of a number of terms, most notably, in the case of an isotropic porous bed, a permeability coefficient and a Forchheimer coefficient. Initial efforts in defining these terms were based on a combination of physical reasoning

\footnotetext{
* Corresponding author.

E-mail address: alessandro.bottaro@unige.it (A. Bottaro).
}

and measurements, leading to expressions known as the KozenyCarman (Kozeny, 1927; Carman, 1937) and the Ergun (Ergun and Orning, 1949) correlations. The first coefficient provides the permeability for the laminar flow of a single-phase fluid through a packed bed of sand grains, as function of the porosity and the diameter of the grains, while the second extends Darcy's law to let the pressure drop depend on two terms, one proportional to the velocity and the second to its square, thus accounting for inertia. These approaches do not consider microstructural or geometrical features of the porous bed, which can render the permeability a tensorial quantity, and are often restricted to simple unidirectional flows. In the present work we are concerned with a transversely isotropic material composed by parallel fibers of circular cross-section, with one axis of symmetry, $\left(0, x_{3}\right)$; in such materials the permeability is a diagonal tensor with the component in the direction parallel to the fibers greater than those along the transverse axes. For such an arrangement we will investigate the effects of both the direction of the forcing pressure gradient and inertia. When the latter effect is present, embodied by a Reynolds number 
$R e_{d}$, based on the mean intrinsic velocity through the medium and the fibers' diameter, exceeding an order one threshold, the permeability is no more simply defined upon geometrical properties. This new permeability, which arises from a well-defined closure problem, is then called apparent permeability.

The influence of the geometry of the solid inclusions has been addressed previously by Yazdchi et al. (2011) for arrays of cylinders in both square and hexagonal (or staggered) patterns, with the cylinders' section which can vary in shape. The results, in the twodimensional and low Reynolds number limits, demonstrate the dependence of the permeability component along the flow direction to both the porosity and the direction of the macroscopic pressure gradient. The direction of the pressure gradient is found to have a weak effect for beds of medium-high porosity $(\varepsilon>0.7)$ and a stronger dependence appears upon the geometry of the solid inclusions.

The influence of the Reynolds number on the permeability and on the Forchheimer correction has been presented in a number of papers. One of the contributions most relevant here is due to Edwards et al. (1990). These authors show that, for arrays of fibers, the apparent permeability decreases with the increase of the Reynolds number, and the rate of this decrease depends on the geometry of the array; also, the Reynolds number is found to have a stronger influence on the apparent permeability when the medium is highly porous. The results of the work by Edwards et al. (1990) agree with those by Zampogna and Bottaro (2016) and with our own work (as shown later), all for the case of cylindrical fibers, although some issues remain on the persistence of steady solutions in the simulations by Edwards et al. (1990) in cases for which a limit cycle should have set in. A fully three-dimensional porous medium, more complex than those discussed so far, has been considered by Soulaine and Quintard (2014), confirming the decreasing trend of the apparent permeability with the Reynolds number.

Another contribution which deserves mention is that by Lasseux et al. (2011); they have computed the permeability tensor for various Reynolds numbers, in a two-dimensional geometry with cylinders of square cross-section. Forcing the flow along the main symmetric directions of the fiber, Lasseux et al. (2011) have identified different regimes:

- a creeping flow regime for $0<R e_{d}<10^{-3}$, without Forchheimer terms;

- a weak inertia regime for $10^{-3}<R e_{d}<1$, with the Forchheimer correction quadratic in $\mathrm{Re}_{d}$;

- a strong inertia regime for $1<R e_{d}<10$, where the Forchheimer correction is linear with the Reynolds number;

- a turbulent regime, for $R e_{d}>10$, with the Forchheimer correction again quadratic with the Reynolds number.

The boundaries between the different regimes are specific to the geometrical arrangements and to the porosities being considered; a step forward in rendering (some of) these boundaries rigorous and independent of the arrangement of the pores, through the definition of a Reynolds number which accounts for a "topological" coefficient, has been recently made by Pauthenet et al. (2017). For the purposes of the present paper, we must retain that Lasseux et al. (2011) have parametrized the Forchheimer correction with the Reynolds number, and have found that the inertial correction is orders of magnitude smaller than the Darcy's term, at least before the turbulent regime sets in. Moreover, Lasseux and co-workers have studied how a Forchheimer tensor, F, depends upon the direction of the macroscopic forcing term with respect to the orientation of the square cross-section of the fibers, for $R e_{d}$ up to 30. It is concluded that a deviation angle, $\gamma$, exists between the direction of the pressure gradient and that of the mean flow, because of the fibers' geometry. The inertial correction is strongly

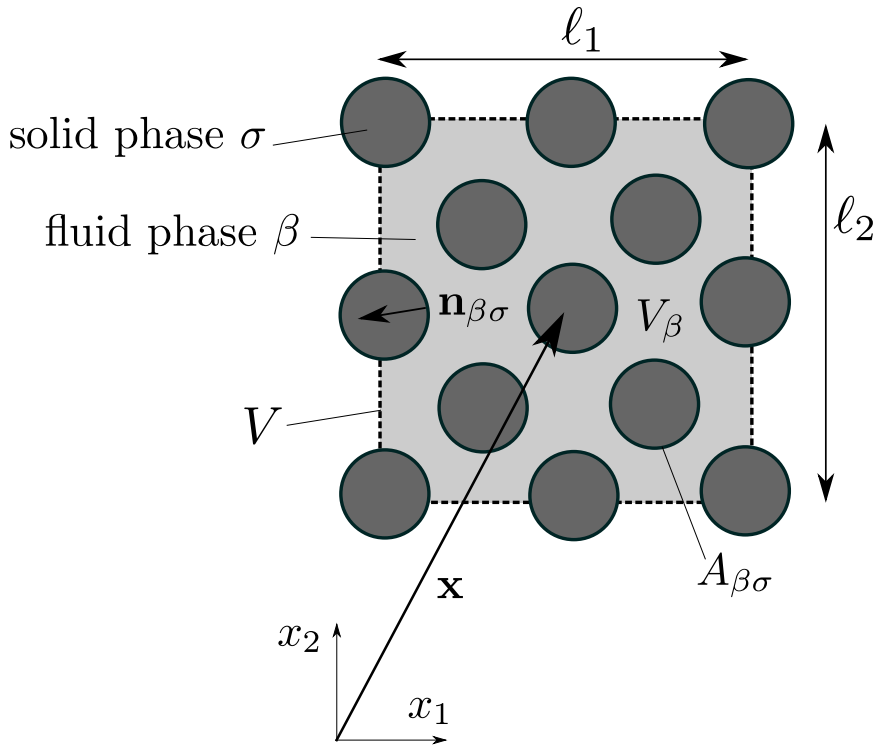

Fig. 1. Illustration of the REV concept.

influenced by the orientation of the driving pressure gradient, and the tensor $\mathbf{F}$ is not symmetric (in fact the off-diagonal components are found to be inversely proportional to the diagonal terms, and symmetric with respect to rotations about the diagonal axis of the square, i.e. the direction at $45^{\circ}$ in the $x_{1}-x_{2}$ plane, cf. Fig. 1 ).

The effect of variations in the forcing angle, with restrictions to angles in the $x_{1}-x_{2}$ plane, is also examined by Soulaine and Quintard (2014) with conclusions in qualitative agreement with those of both the contribution just cited and our results described further below. In all cases, the off-diagonal components of the apparent permeability tensor are small and the diagonal components display but a small variation upon rotation of the driving pressure gradient.

As already anticipated, this work investigates how the direction of the macroscopic pressure gradient, the porosity and the Reynolds number can modify the Darcy and Forchheimer closures arising from a volume-averaged model of a fibrous porous medium. We will consider a three-dimensional unit cell for the microscopic model (such a unit cell is sometimes denoted REV, for Representative Elementary Volume), with a generic forcing whose direction is defined by two Euler angles. Given the formidable space of parameters, some representative results are first shown and discussed. Response surfaces in the space of parameters are then identified by the use of a metamodel based on kriging interpolation. For the sake of space, only the first diagonal component of the apparent permeability tensor is discussed in detail in the paper; however, all components have been computed. They represent an extremely useful data base which we are now in the process of using in macroscopic simulations of flows through bundles of fibers of varying orientation and density.

\section{The volume-averaged Navier-Stokes (VANS) method}

\subsection{A brief description of the method}

The system under investigation consists of an incompressible Newtonian fluid which flows through a rigid porous medium. In the following, the subscript $\beta$ is used to indicate the fluid phase while $\sigma$ is adopted for the solid phase. The governing equations valid at the microscale for the flow through the pores are:

$\frac{\partial \mathbf{v}_{\beta}}{\partial t}+\mathbf{v}_{\beta} \cdot \nabla \mathbf{v}_{\beta}=-\frac{1}{\rho_{\beta}} \nabla p_{\beta}+v_{\beta} \nabla^{2} \mathbf{v}_{\beta}+\mathbf{f}$, 
$\nabla \cdot \mathbf{v}_{\beta}=0$

where $\mathbf{v}_{\beta}, p_{\beta}, \rho_{\beta}$ and $v_{\beta}$ stand, respectively, for the velocity, the pressure, the density and the kinematic viscosity of the fluid. The right-hand side term, $\mathbf{f}$, is a force (per unit mass) which drives the fluid motion and can be interpreted as the macroscopic pressure gradient acting on the system.

The concept of REV of the porous medium is classically introduced in the framework of the VANS approach. An example of REV is depicted on Fig. 1, together with relevant notations (volume shape and size, indication of the fact that the normal unit vector is directed from the fluid to the solid phase, centroid $\mathbf{x}$ of the REV). The REV represents the domain over which the microscopic problem is solved; its size is defined so as to capture all the microscopic features of the flow. As a rule of thumb, the REV is the smallest fluid domain over which periodic boundary conditions can be applied.

In the computational domain, any flow variable $\psi$ can be decomposed into an intrinsic average part $\langle\psi\rangle^{\beta}$ plus a perturbation $\tilde{\psi}$, as:

$\psi=\langle\psi\rangle^{\beta}+\tilde{\psi}$.

The intrinsic average is defined with an integration carried out only on the fluid phase (Whitaker, 2013):

$\left\langle\psi_{\beta}\right\rangle^{\beta}=\frac{1}{V_{\beta}} \int_{V_{\beta}} \psi_{\beta}(\mathbf{x}) d V_{\beta}$.

Applying such an operator to Eqs. (1) and (2), and following Whitaker (1996) we have:

$$
\begin{aligned}
\frac{\partial\left\langle\mathbf{v}_{\beta}\right\rangle^{\beta}}{\partial t}+\left\langle\mathbf{v}_{\beta}\right\rangle^{\beta} \cdot \nabla\left\langle\mathbf{v}_{\beta}\right\rangle^{\beta}= & -\frac{1}{\rho_{\beta}} \nabla\left\langle p_{\beta}\right\rangle^{\beta}+v_{\beta} \nabla^{2}\left\langle\mathbf{v}_{\beta}\right\rangle^{\beta}+\mathbf{f}+ \\
& +\frac{1}{V_{\beta}} \int_{A_{\beta \sigma}}\left(-\frac{\tilde{p}_{\beta}}{\rho_{\beta}} \mathbf{I}+v_{\beta} \nabla \tilde{\mathbf{v}}_{\beta}\right) \cdot \mathbf{n}_{\beta \sigma} d A,
\end{aligned}
$$

$\nabla \cdot\left\langle\mathbf{v}_{\beta}\right\rangle^{\beta}=0$,

upon neglecting in Eq. (4) the sub-REV-scale dispersion term (linked to $\left\langle\tilde{\mathbf{v}}_{\beta} \tilde{\mathbf{v}}_{\beta}\right\rangle^{\beta}$ ) which is often small in porous media flows (Breugem, 2005).

The surface integral term in Eq. (4) represents the drag (per unit mass) due to surface forces at the fluid-solid interface of the medium. It is called the Darcy-Forchheimer microscale force, $\mathbf{F}^{m}$. The equations are however often to be solved at the macroscale, so that a macroscale force model, $\mathbf{F}^{M}$, must be used to replace $\mathbf{F}^{m}$ in the governing equation. Such a model is often based on a permeability tensor, $\mathbf{K}$, and a Forchheimer tensor, $\mathbf{F}$, and reads:

$\mathbf{F}^{M}=-v_{\beta} \varepsilon \mathbf{K}^{-1}(\mathbf{I}+\mathbf{F})<\mathbf{v}_{\beta}>^{\beta}$,

so that the system is closed by imposing

$\mathbf{F}^{m}=\mathbf{F}^{M}$.

The drag force $\mathbf{F}^{m}$ computed by direct numerical simulations (DNS) with account of all individual pores will be later compared to the model based on the permeability and Forchheimer tensors (whose equations are given below). This is a useful exercise to demonstrate consistency of the approach and accuracy of the numerical simulations; it does nothing else since, as briefly described below, to derive the Forchheimer tensor the microscopic velocity field must be known anyhow. Nonetheless, knowledge of the behaviour of these tensors (or, equivalently, of the related apparent permeability) might prove both useful and instructive, in particular should one wish to extend the range of applicability of the model to cases for which the microscopic solution is not available.

The core of the VANS approach consists in the identification of the permeability and Forchheimer tensors. This problem, referred to as the closure problem, is discussed at length by Whitaker (1986, 1996). He derives two partial differential equation systems, the first valid in the zero Reynolds number limit (system (8) below), while the second applies when inertial terms are not negligible (system (10)).

In the first system of equations a three-component vector $\mathbf{d}$ and a $3 \times 3$ tensor $\mathbf{D}$ are introduced. This system can be divided into three separate independent problems which resemble a forced Stokes problem where each component of $\mathbf{d}$ and the corresponding row of $\mathbf{D}$ play, respectively, the role of a pressure and a velocity field. Together with the periodic boundary conditions, the problem reads:

$$
\left\{\begin{array}{l}
0=-\nabla \mathbf{d}+\nabla^{2} \mathbf{D}+\mathbf{I}, \\
\nabla \cdot \mathbf{D}=0, \\
\mathbf{D}=0 \quad \text { on } \quad A_{\beta \sigma}, \\
\mathbf{d}\left(\mathbf{x}+\ell_{i}\right)=\mathbf{d}(\mathbf{x}), \quad \mathbf{D}\left(\mathbf{x}+\ell_{i}\right)=\mathbf{D}(\mathbf{x}) \quad i=1,2,3 .
\end{array}\right.
$$

The permeability tensor is found by applying the intrinsic average on the $\mathbf{D}$ tensor and multiplying by the porosity $\varepsilon=\frac{V_{\beta}}{V}$, i.e. $\mathbf{K}=$ $\varepsilon<\mathbf{D}>\beta$. In the Stokes regime, it is

$\mathbf{F}^{M}=-v_{\beta} \varepsilon \mathbf{K}^{-1}<\mathbf{v}_{\beta}>^{\beta}$.

The second closure problem differs from the first only for the presence of a linearised convective term in which the microscopic velocity obtained from the DNS, $\mathbf{v}_{\beta}$, is used as an input. This of course implies knowledge of the microscopic velocity field. A Oseen-like approximation which relaxes this constraint has been proposed by Zampogna and Bottaro (2016).

The new unknowns are a vector and a tensor called, respectively, $\mathbf{m}$ and $\mathbf{M}$, with the same meanings of $\mathbf{d}$ and $\mathbf{D}$. The system reads:

$$
\left\{\begin{array}{l}
\frac{1}{v_{\beta}} \mathbf{v}_{\beta} \cdot \nabla \mathbf{M}=-\nabla \mathbf{m}+\nabla^{2} \mathbf{M}+\mathbf{I}, \\
\nabla \cdot \mathbf{M}=0, \\
\mathbf{M}=0 \quad \text { on } \quad A_{\beta \sigma}, \\
\mathbf{m}\left(\mathbf{x}+\ell_{i}\right)=\mathbf{m}(\mathbf{x}), \quad \mathbf{M}\left(\mathbf{x}+\ell_{i}\right)=\mathbf{M}(\mathbf{x}) \quad i=1,2,3 .
\end{array}\right.
$$

The average of the tensor $\mathbf{M}$ multiplied by the porosity is the apparent permeability, $\mathbf{H}=\varepsilon\langle\mathbf{M}\rangle^{\beta}$. When inertia is important Eq. (6) can be written as

$\mathbf{F}^{M}=-v_{\beta} \varepsilon \mathbf{H}^{-1}<\mathbf{v}_{\beta}>^{\beta}$,

as shown by Whitaker (1996).

Two remarks are in order at this point. First, the equations in the closure problem (10) are time-independent because the microscopic velocity $\mathbf{v}_{\beta}$ is a solution of a stationary DNS. Thus, the Reynolds number should be sufficiently small for unsteady effects not to be present. Should the wake behind a solid inclusion display regular or irregular temporal oscillations, the equations of system (10) may be used, as an approximation, by replacing the instantaneous velocity in the REV with its time-averaged distribution. This case is however not of present concern. Secondly, the closure problems reflect the structure of the solution of the two systems (8) and (10). In particular, the solution of (8) depends only on the geometry of the porous medium so that the permeability tensor $\mathbf{K}$ is symmetric. This is not the case for $\mathbf{H}$, because of the effect of the microscopic velocity amplitude and direction. Clearly, the solution of system (8) tends to that of (10) when $R e_{d}=\frac{\left\langle\mathrm{v}_{\beta}\right\rangle^{\beta} d}{v_{\beta}} \rightarrow 0$, 

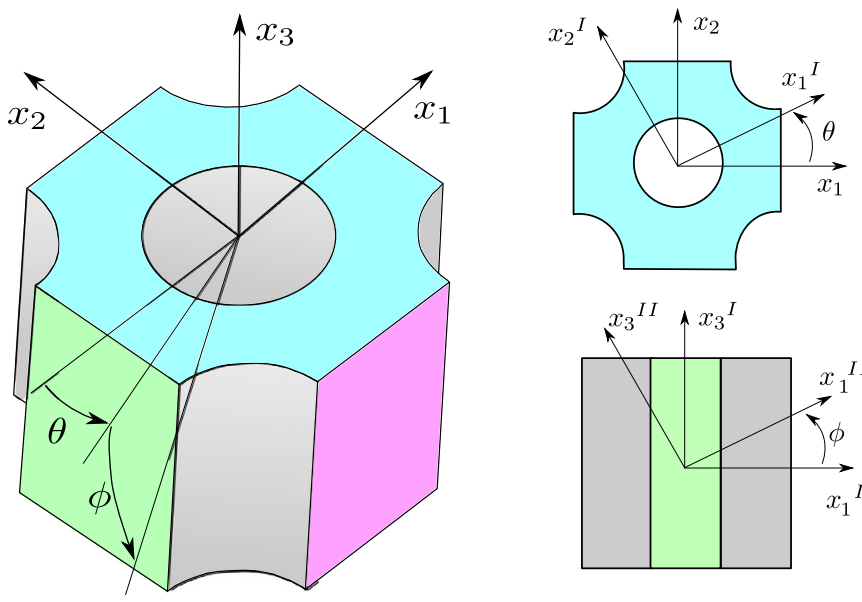

Fig. 2. REV for the fiber geometry investigated.

with $\mathrm{v}_{\beta}$ the amplitude of the intrinsic velocity and $d$ the diameter of the fiber.

\section{Validation and setup}

In this section the numerical methodology, the parameters, the setup and the validation for some reference cases are given.

\subsection{Computational domain}

The geometry used for the base REV is shown in Fig. 2: a cylindrical inclusion is present at the centre of the REV and four quarters of cylinders are situated at the corners. The lateral length of the cubic envelop is $\ell$, which is used as length scale for the microscopic problem; the diameter $d$ of the cylinders is adapted as a function of the desired porosity $\varepsilon$, ratio between the fluid volume over the total REV volume $\left(\ell^{3}\right)$. The forcing term $\mathbf{f}$ of the DNS is a vector whose direction is defined by two Euler angles, with rotations of the form: $\theta \mathbf{e}_{\mathbf{3}}+\phi \mathbf{e}_{\mathbf{2}}{ }^{I}$ (cf. Fig. 2). Its amplitude is set a priori and is connected to the Reynolds number, $R e_{d}$ which results from the calculations once the mean velocity is evaluated.

\subsection{Numerical setup}

The numerical simulations of the full Navier-Stokes Eqs. (1)-(2) in the REV have been carried out with the open-source code OpenFOAM (Weller et al., 1998), based on a finite volume discretization with a colocated arrangement for the unknowns. The standard solver icoFoam (incompressible Navier-Stokes) has been modified in order to include a constant pressure gradient acting as a forcing term $\mathbf{f}$ in Eq. (1). The coupling between the velocity and the pressure equations is based on the pressure implicit split operator referred to as the PISO algorithm. The time derivative term is discretized using the second order backward Euler scheme and all the spatial terms use a second-order central difference stencil based on Gauss finite volume approach. The velocity system is solved with a preconditioned bi-conjugate gradient iterative solver with the tolerance on the velocity residuals set to $10^{-8}$, associated to a diagonal incomplete lower upper pre-conditioner. The pressure equation is solved with a geometric-algebraic multigrid algorithm associated to a Gauss-Seidel smoother and the tolerance on the pressure residuals is taken equal to $10^{-6}$. Periodic boundary conditions are applied to all fields (velocity and pressure) on all fluid boundaries along the three directions, and the no-slip condition is imposed on the surface of the solid inclusions. The time step $\Delta t$ is automatically determined to ensure that the maximum Courant number,

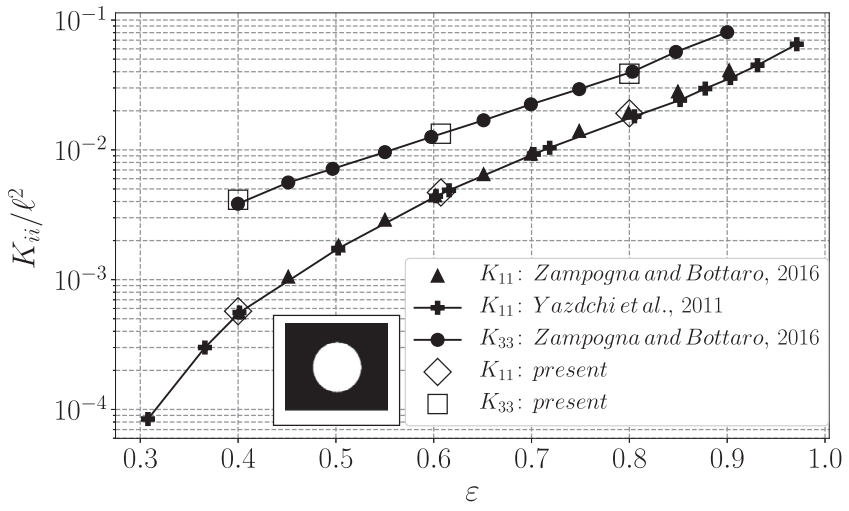

Fig. 3. Permeability versus porosity for a square arrangement of cylinders. The scaling of the permeability is $\ell^{2}$ and is explicitely indicated in the vertical axis.

Table 1

Relatives errors in the effective permeability validation.

\begin{tabular}{llll}
\hline$\varepsilon$ & $\begin{array}{l}\text { Regular REV } \\
e_{r_{11}}\end{array}$ & $e_{r_{K_{33}}}$ & $\begin{array}{l}\text { Staggered REV } \\
e_{r_{K_{11}}}\end{array}$ \\
\hline 0.4 & $2.34 \%$ & $3.22 \%$ & $4.13 \%$ \\
0.6 & $1.58 \%$ & $0.38 \%$ & $2.03 \%$ \\
0.8 & $4.10 \%$ & $2.03 \%$ & $2.17 \%$ \\
\hline
\end{tabular}

Co, respects the condition: $C_{0}=v_{\beta} \Delta t / \Delta s / 1 / 2$, in which $v_{\beta}$ is the modulus of the local velocity on the REV and $\Delta s$ is the local grid spacing in the direction of $\mathbf{v}_{\beta}$. Co is basically the ratio between the fluid speed and the velocity to propagate information through the mesh and the condition $C_{0}<1 / 2$ is found to be sufficient to have a stable solver.

The convergence of the solutions is assessed on the basis of a Richardson extrapolation which employs the results obtained on successively refined grids (with up to 1.5 million cells in the finer grid used). The mesh convergence analysis is described in Appendix A.

\subsection{Validation on two different configurations}

The results published in the literature by Zampogna and Bottaro (2016) and Yazdchi et al. (2011) are now used to validate both the methodology and our choice of the computational parameters. In the cited papers, three-dimensional computations of the permeability components in different cells geometries are presented.

Fig. 3 displays the comparison for a cell with a square arrangement of the fibers; here the permeability is evaluated along the two principal directions, $x_{1}$ and $x_{3}$. To quantify the accuracy of the results we introduce a relative error on the permeability components, defined as $e_{r_{K_{i i}}}=\frac{\left|K_{i i}{ }^{\text {present }}-K_{i i}^{\text {literature }}\right|}{K_{i i}^{\text {literature }}}$ and reported in Table 1. Good agreement is found with the published results: the relatives errors in all the cases tested is always below 5\%. Fig. 4 shows a similar comparison for a staggered arrangement of the inclusions in the unit cell. In this case the section of the cell is rectangular. The agreement for the only permeability component available in the literature is again satisfactory. Finally, to check the correct implementation of the closure model (10) it is important to verify the equality (7) between the amplitude $F^{M}$ of the macroscopic force and its microscopic counterpart obtained through an integration of the DNS fields over the solid boundaries of the inclusions in the REV. Fig. 5 shows a plot of the relative error between these two forces, i.e. $\frac{\left\|F^{M}-F^{m}\right\|}{\left\|F^{m}\right\|}$, as function 


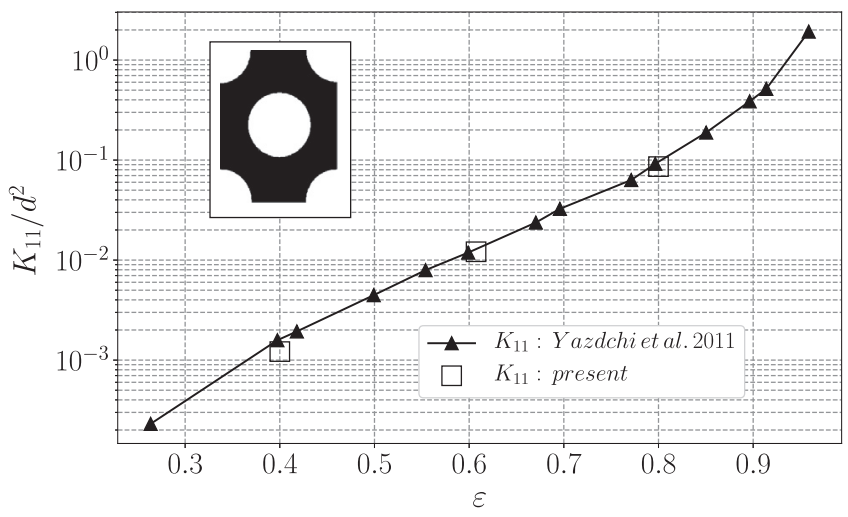

Fig. 4. Permeability versus porosity for a staggered arrangement of cylinders. Note that the permeability component is here scaled with $d^{2}$.

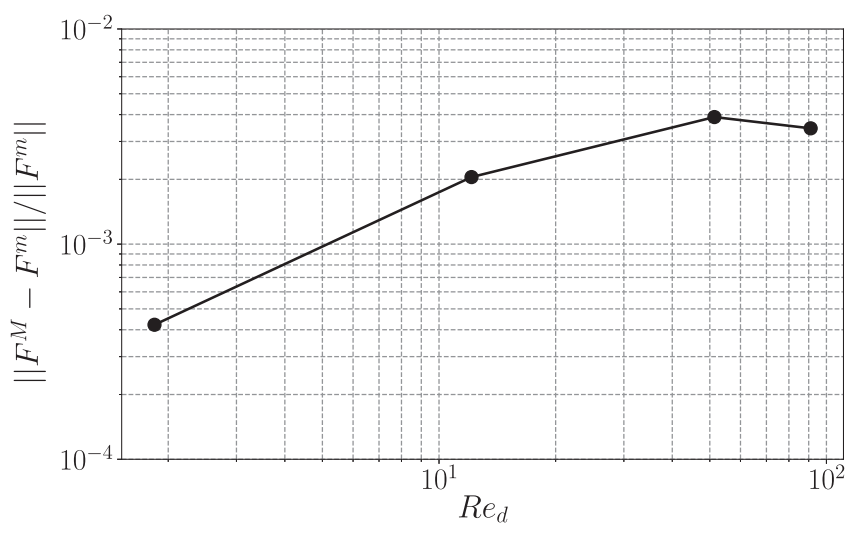

Fig. 5. Relative error between the microscopically computed forces along the $x_{1}$ direction and those arising from the Darcy-Forchheimer model; $\varepsilon=0.8$ for the REV in the staggered arrangement of Yazdchi et al. (2011).

of the Reynolds number. We consider the successful comparison displayed in Fig. 5 as the conclusive demonstration of the validity of the approach described here. We have nonetheless carried out the same verification displayed in Fig. 5 for most of the simulations described in the following, to our satisfaction.

\subsection{Tests with larger REV's}

Since the REV is the unit cell within the porous medium over which average quantities of the VANS are computed, it is important to choose its dimensions appropriately in the inertial regime for, if the REV is too small, it might be easy to miss crucial features of the wakes. For example, to predict the critical Reynolds number, $R e_{c}$, of the first Hopf bifurcation, a REV containing at least three solid inclusions in the direction of the mean pressure gradient is necessary in the configuration used by Agnaou et al. (2016). Among the results reported, it is found that, for a fixed REV size, the error committed in the evaluation of the critical Reynolds number increases with the porosity. This same error is considerably reduced when the mean pressure gradient angle is $\theta=45^{\circ}$. Thus, the choice of the number of inclusions in a REV is a task not to be overlooked, and the final choice must account for the porosity, the direction of the pressure gradient and the microscopic Reynolds number.

Here, the influence of the numbers of inclusions present in a REV is assessed by focussing only on the velocity components after averaging over the REV. The unit cubic cell of side $\ell$ is used as reference: starting from this, two additional REV's are built, as shown in Fig. 6. The first one is doubled in both the $x_{1}$ and $x_{2}$ directions and the case tested numerically is characterised by $\theta=0, \phi=0$

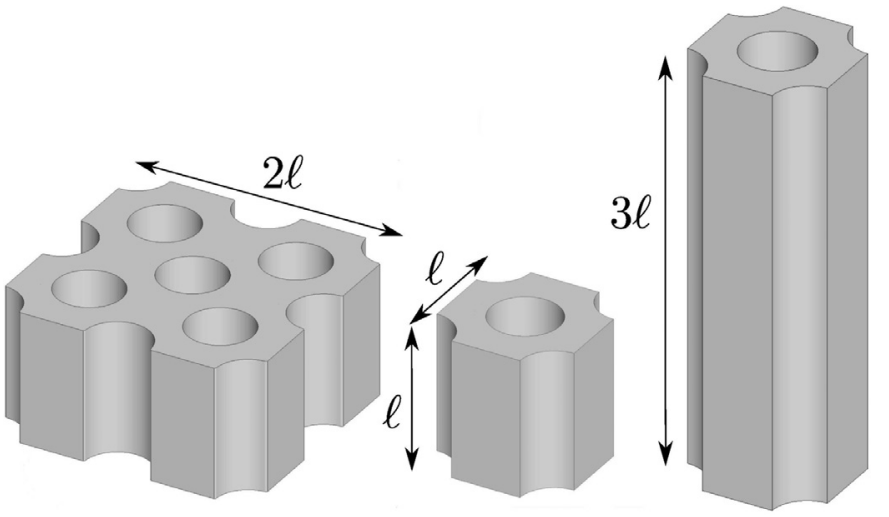

Fig. 6. REV configurations. Left: $2 \times 2 \times 1$ arrangement; centre: $1 \times 1 \times 1$ arrangement (reference); right $1 \times 1 \times 3$ arrangement.

(i.e. the forcing pressure gradient is directed along $x_{1}$ ), porosity $\varepsilon=0.6$ and $R e_{d}=50$. The second REV configuration is a composition of 3 reference REVs on top of one another along $x_{3}$, with the parameters set to $\theta=45^{\circ}, \phi=45^{\circ}, \varepsilon=0.6$ and $R e_{d}=100$.

For both these test cases, no appreciable differences, neither in the mean velocity nor in the forces on the fibers, have been observed, with relative errors on the mean velocity with respect to the reference case which remain below $2 \%$. We take this as sufficient evidence to use, in the following, only the reference cubic REV of side equal to $\ell$, with the understanding that only cases with a microscopic Reynolds number up to around 100 can be considered.

\section{Microscopic solutions}

In this section, some local microscopic fields computed with direct numerical simulations are shown, together with components of the intermediate tensor $\mathbf{M}$ coming from the numerical solution of the closure equations (10).

In Fig. 7 (top row) the local $x_{1}$ velocity component is drawn for the two-dimensional flow when $\varepsilon=0.6$, for three Reynolds numbers, to cover the transition from the Stokes to the inertial regime. In all plots, the velocities are rendered non-dimensional by the corresponding value of $\frac{H_{11}}{v_{\beta}}\|\mathbf{f}\|$, where $H_{11}$ is the first component of the tensor $\mathbf{H}$. When inertia is absent, the flow has a central symmetry; by increasing the Reynolds number, only the symmetry with respect to the $x_{1}$ axis is maintained ( $x_{1}$ is the direction of the forcing pressure gradient), with the wake's length which increases with $R e_{d}$. When $R e_{d}$ is of order 100 the wake spreads to the downstream boundary of the REV, re-entering, because of periodicity, at the upstream side. This $R e_{d}$ represents the upper limit of validity for the cubic unit cell of side $\ell$; larger values of $R e_{d}$ could only be investigated with longer/larger/thicker REV's.

The non-dimensional microscopic $M_{11}$ fields for the same parameters are displayed in Fig. 7 (mid row). All values in the figures arise from scaling $\mathbf{M}$ with $\ell^{2}$. Visually, these local fields are strongly correlated to the local streamwise velocity component in the whole $R e_{d}$ range. This is not unexpected since the local velocity drives the convective term of system (10). The central symmetry of all components of $\mathbf{M}$ in the Stokes regime is coupled to the rotational invariance of the apparent permeability tensor in twodimensional flows.

The effect of varying the porosity is shown in Fig. 7 (bottom row) where $\varepsilon$ is taken equal to 0.4 . Even at such a low porosity the stretching of the wake can be noticed, and it increases with $R e_{d}$. Interestingly, this effect is milder when the forcing is inclined by an angle $\phi$, since the tighter packing of the inclusions causes 

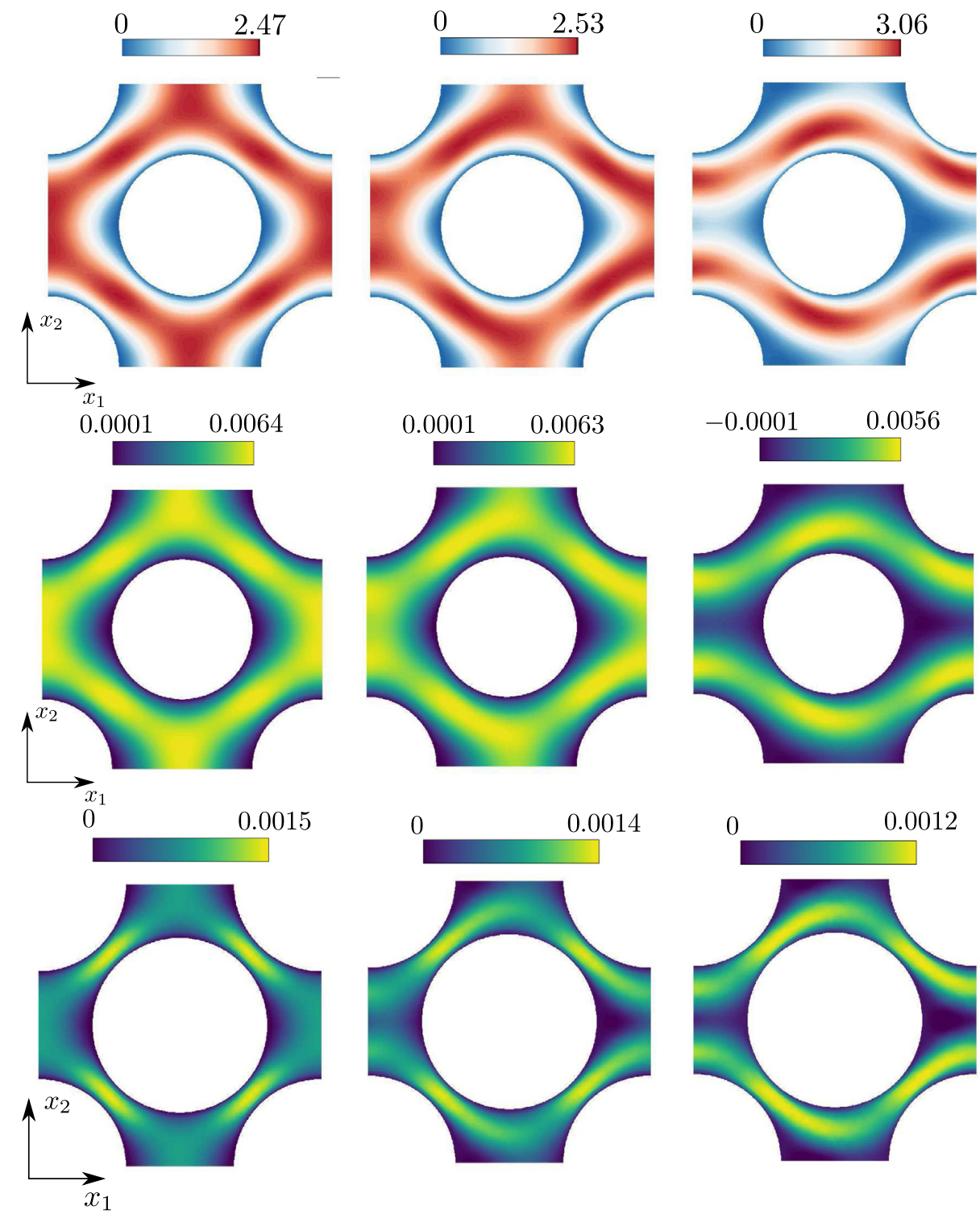

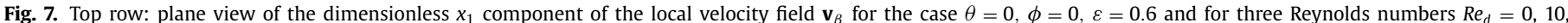

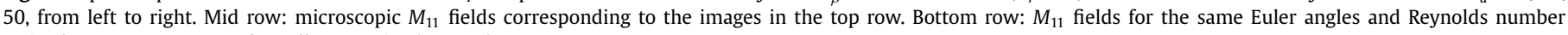
as in the top two rows, and smaller porosity $(\varepsilon=0.4)$.

a strong deviation of the mean flow along the axis of the fiber. In this case, $M_{11}$ and $M_{22}$ behave very similarly to the case $\phi=90^{\circ}$.

Another interesting point emerges by inspection of Fig. 8 where two off-diagonal components of $\mathbf{M}$ are shown for two porosity values; the first image (left frame) represents a plane flow in the Stokes regime while the second is the plane cut of a threedimensional solution in the inertial regime. Positive and negative values of the microscopic fields can be seen in both images but, once averaging is applied over the REV, the resulting permeability component is very close to zero (in fact, exactly equal to zero in the Stokes case). This same features occurs for all off-diagonal terms in all cases examined, so that, within the current range of Reynolds numbers, the apparent permeability tensor is, to a good approximation, diagonal ${ }^{1}$

A three-dimensional case is shown in Fig. 9, where all the nonzero terms of the $\mathbf{M}$ tensor are plotted for a porous structure with

\footnotetext{
${ }^{1}$ In fact, there are always at least two orders of magnitude differences between the diagonal and the off-diagonal components. While the latter should not, in prin-
}

$\varepsilon=0.6$. The components shown are $M_{11}, M_{22}, M_{33}, M_{12}$ and $M_{21}$, while $M_{i 3}$ and $M_{3 j}$ are not plotted because they are identically zero to machine accuracy. Distinct features are visible in each image; in particular, in the last frame the $M_{33}$ microscopic component displays a low wavelength structure along the cylinder's axis. Increasing the dimensions of the REV along $x_{3}$ does not alter such a structure, i.e. the $\ell^{3}$ domain chosen with its periodic boundary conditions does not filter out significant high wave-numbers of the flow. We further note that the tensor $\mathbf{M}$ is not symmetric in this case since each off-diagonal component represents the solution of the closure problem in a specific direction (first index of the field) and the forcing term acts orthogonally to it (second index of the field). Once averaged over the REV it is found that both $H_{12}$ and $H_{21}$ are very close to zero.

ciple, be ignored, we will focus attention here only on the dominant terms of the permeability tensor. 

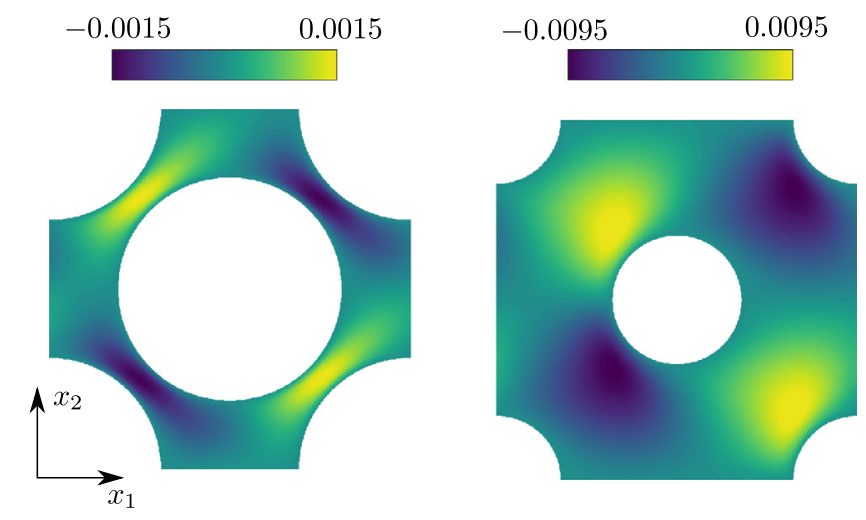

Fig. 8. Right: Non-dimensional $M_{21}$ field for $\theta=0, \phi=0, R e_{d}=10, \varepsilon=0.8$, left: Non-dimensional $M_{12}$ field for $\theta=22.5^{\circ}, \phi=45^{\circ}, R e_{d}=50, \varepsilon=0.4$.

\section{Table 2}

Directions of the forcing tested and property of the solutions.

\begin{tabular}{llll}
\hline Index & $\theta$ & $\phi$ & Field properties \\
\hline 1 & $0^{\circ}$ & $0^{\circ}$ & 2D symmetric \\
2 & $22.5^{\circ}$ & $0^{\circ}$ & 2D non-symmetric \\
3 & $0^{\circ}$ & $45^{\circ}$ & 3D symmetric \\
4 & $22.5^{\circ}$ & $45^{\circ}$ & 3D non-symmetric \\
5 & - & $90^{\circ}$ & 3D symmetric \\
\hline
\end{tabular}

\section{The apparent permeability tensor}

In this section the variations of the diagonal components of the permeability tensor $\mathbf{H}$ are discussed as function of the direction of the mean forcing, the Reynolds number and the porosity. As stated previously, the Reynolds number $R e_{d}$ ranges from 0 to approximately 100 in order to capture phenomena associated with inertia; the cases considered never lead to unsteady signals. The porosity parameter $\varepsilon$ is set to either 0.4 (low porosity), 0.6 (medium) or 0.8 (high).

The forcing direction is defined by the Euler angles and all the configurations considered in this section are summarized in Table 2; the choice has been made to explore a reasonably large range of parameters, with both two-dimensional and three- dimensional flows characterized by symmetric and asymmetric patterns.

Let us briefly recall the methodology. First, a DNS is carried out to compute the microscopic flow. Then the closure problem is solved for the tensor M. Finally, each component of the apparent permeability $\mathbf{H}$ is obtained by averaging (Eq. (3)). The results are collected in Figs. 10, 11 and 12, showing the variation of the diagonal components of $\mathbf{H}$.

In the left column of each figure we focus on the low- $R e_{d}$ regime $\left(0<R e_{d}<2\right)$, while in the right column the effect of inertia can be assessed. As expected, when $R e_{d}$ is small the apparent permeability is quasi-Reynolds-number-independent (and can be approximated well by the true permeability). As the Reynolds number increases above a few units, inertial effects grow in importance yielding typically a monotonic decrease of all components of $\mathbf{H}$, aside from case indexed $5\left(\phi=90^{\circ}\right)$ for which the flow remains aligned with the cylinder's axis. In case 5 the microscopic flow solution is invariant with $x_{3}$ and does not change with $R e_{d}$ in the range considered, so that $\mathbf{H}$ is a constant tensor. When the porosity is large all components show a similar behaviour irrespective of the forcing angle (except, clearly, case 5).

Differences start appearing at $\varepsilon=0.6$; the two cases with $\phi=$ $0^{\circ}$ (index 1 and 2 ) behave similarly, and so do the two cases indexed 3 and 4 (with $\phi=45^{\circ}$ ). This seems to suggest a weaker effect of $\theta$ on the permeability components. For even smaller poros-

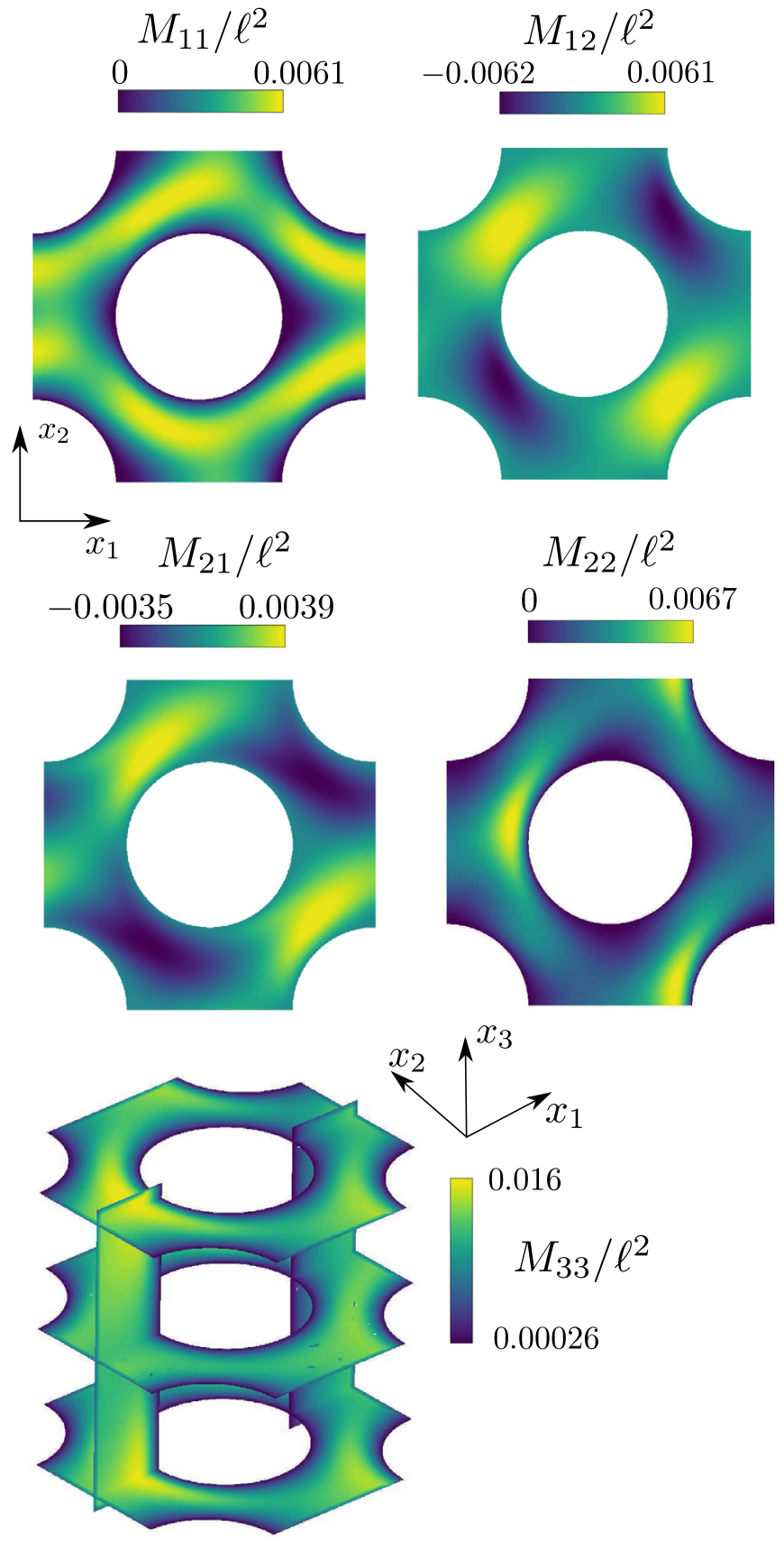

Fig. 9. Non-dimensional $\mathbf{M}$ components fields for the case $\theta=22.5^{\circ}, \phi=$ $45^{\circ}, \operatorname{Re}_{d}=50, \varepsilon=0.6$.

ity $(\varepsilon=0.4$ ), the blockage effect caused by the inclusions produces the unexpected behaviour displayed in Fig. 12. When the flow is purely two-dimensional (cases 1 and 2), variations in the Reynolds number affect $\mathbf{H}$ significantly; when a pressure gradient along $x_{3}$ is present the strong packing of the fibers constrain the fluid to flow prevalently along the fibers' axis, and the apparent permeability is almost $R e_{d}$-independent. When assessing variations in $H_{j j}$ for this case, attention should also be paid to the fact that the permeability is now at least one order of magnitude smaller than in the previous cases so that variations of the diagonal components shown in Fig. 12 are tiny in absolute terms. This is related to the fact that the inverse of the permeability plays the role of a drag coefficient in the macroscopic expression of the force (cf. equation (6)). In other 

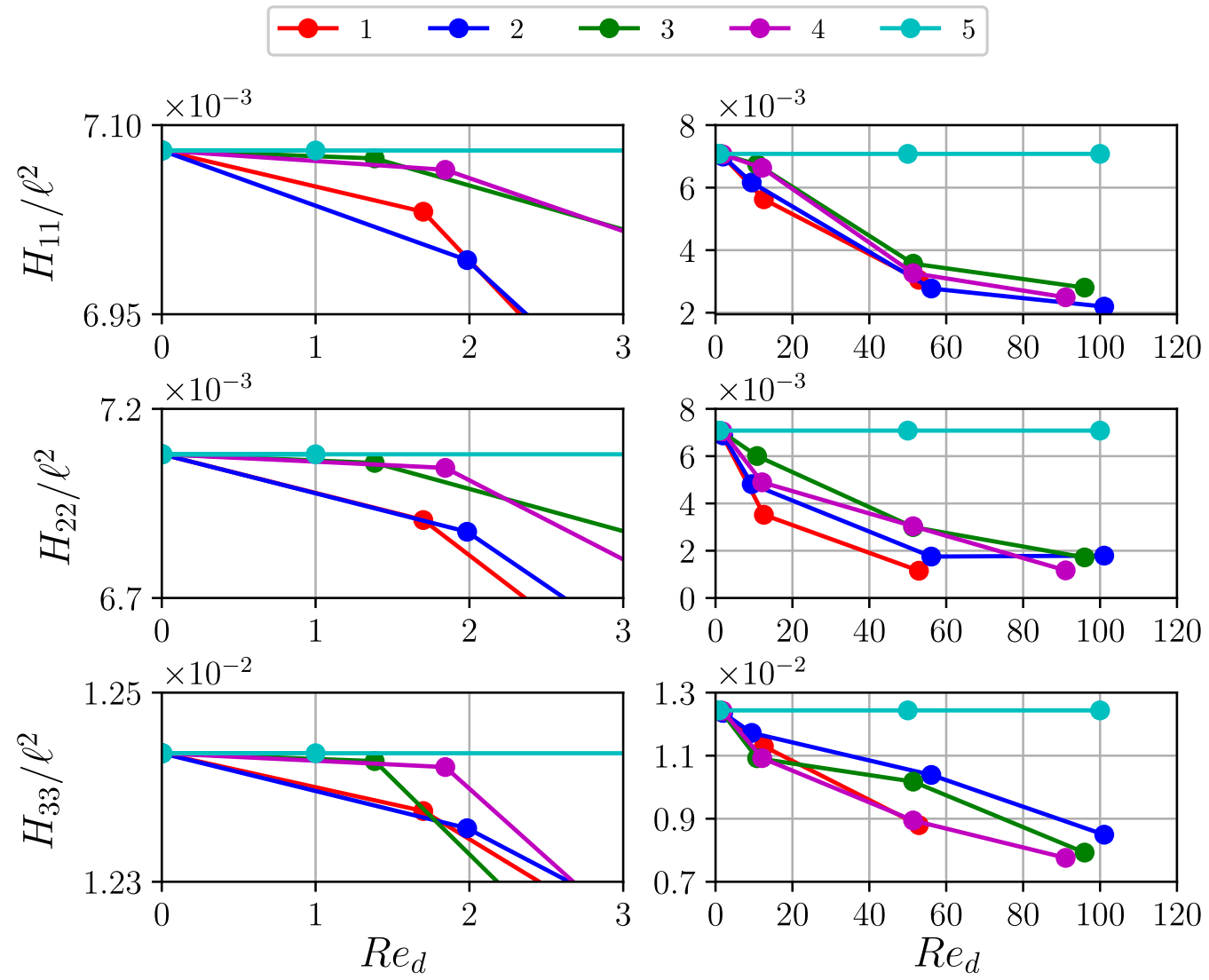

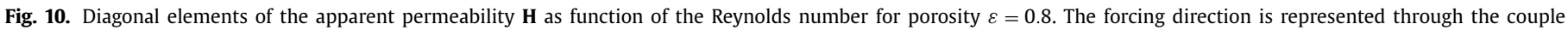
of Euler angles $(\theta, \phi)$ (cf. Table 2 for the case index). Left column: low-Re $e_{d}$ regime; right column: inertial regime.

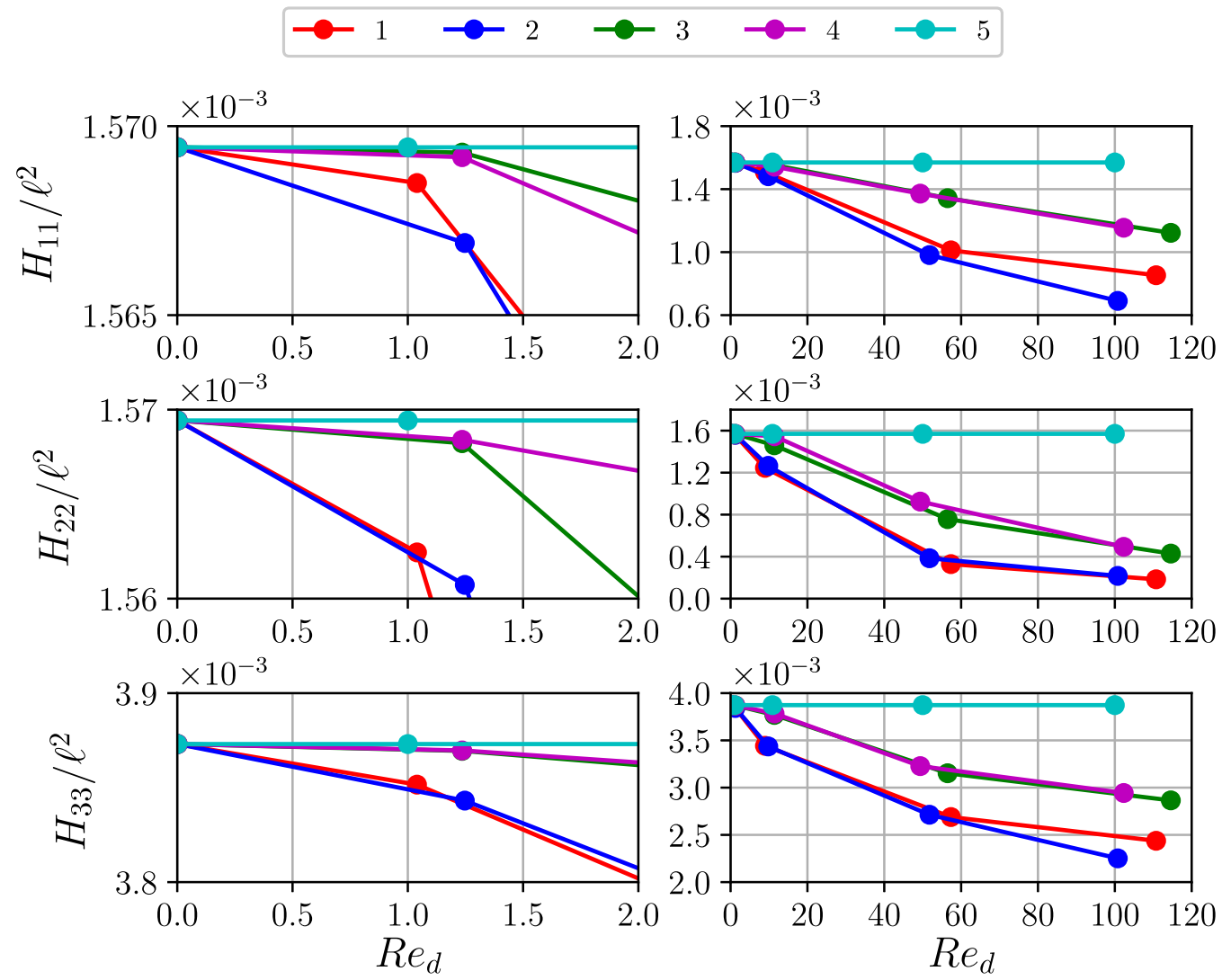

Fig. 11. Same as Fig. 10 with porosity $\varepsilon=0.6$. 


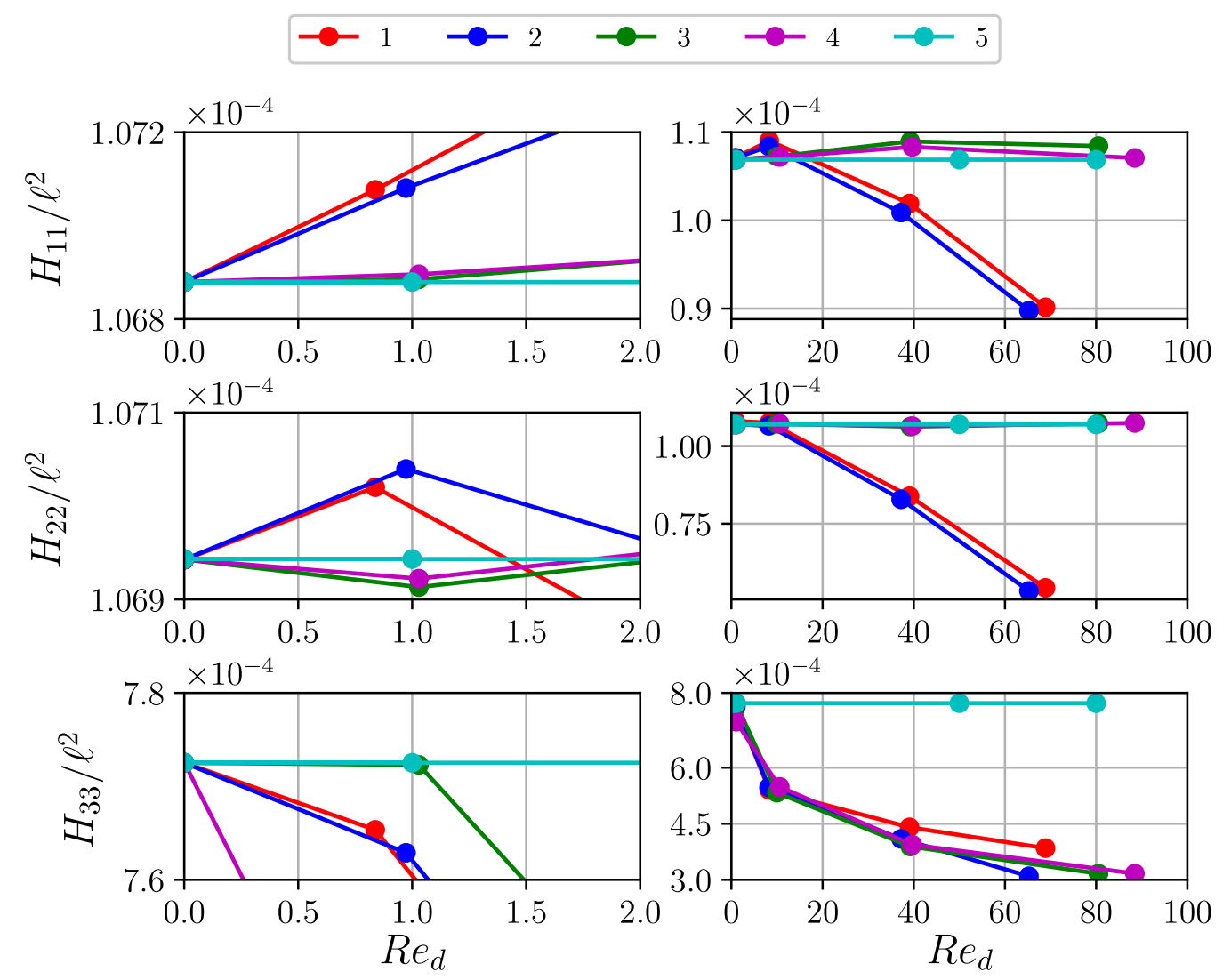

Fig. 12. Same as Fig. 10 with porosity $\varepsilon=0.4$.

words, materials with lower porosity offer larger resistance to the motion of the fluid.

Applying the intrinsic average operator to the off-diagonal components of the tensor $\mathbf{M}$ results in terms that are negligible with respect to their diagonal counterparts, and these results are true for all the parameters considered. This means that there is a very weak coupling between the principal directions of the fiber. The directional decoupling and the diagonal property of the apparent permeability tensor have also been computationally demonstrated on a completely different REV geometry by Soulaine and Quintard (2014). Conversely, Lasseux et al. (2011) have carried out a two-dimensional study with fibers of square cross-section, finding that the off-diagonal terms are non-negligible and only about one order of magnitude smaller than the diagonal components. This result is a consequence of the non-rotationally-invariant geometry considered. The present work and the two articles just cited suggest that the diagonal property of the tensor $\mathbf{H}$ is more closely associated to the geometry of the porous material than to the flow regime, at least in the range of $R e_{d}$ considered.

\section{A metamodel for $\mathrm{H}$}

Sections 4 and 5 have shown how the apparent permeability depends on the two Euler angles, the Reynolds number and the porosity. The space of parameters is formidable and the results found so far are not sufficient to treat, for example, cases characterized by multiple inclusions' sizes and orientations in different regions of the domain, or cases involving a poroelastic medium, with temporally and spatially varying porosity, flow direction and local Reynolds number. The complete solution of the closure problem for a single set of parameters takes approximately $4 \mathrm{CPU}$ hours on our two-processor Intel(r) IVYBRIDGE $2.8 \mathrm{Ghz}$, each with
10 cores and 64 GB of RAM, so that a complete parametric study is, to say the least, unpractical. In view of this, the construction of a metamodel capable to provide a full characterisation of the permeability as a function of all parameters is a worthy endeavor. We have tested several surrogate models, before eventually settling on the kriging approach (Kleijnen, 2017) described in Appendix B .

The metamodel provides a scalar function (for each term of the $\mathbf{H}$ tensor) defined in a four-dimensional space. In each of the following figures two parameters are fixed and the response surface is displayed as function of the remaining two, focussing on the $H_{11}$ component. The other diagonal components of the apparent permeability tensor behave in a similar fashion and will not be shown for brevity. All the results of the metamodel are, however, available on: https://github.com/appanacca/porous_solvers_OF.git.

In Fig. 13 the angle $\phi$ is fixed to zero, and the isolines display $H_{11}$ as function of the angle $\theta$ and of the Reynolds number, $R e_{d}$, for three values of the porosity. The white square symbols indicate the samples used to build the metamodel. The maximum value of each surface is always found for $R e_{d}$ equal to zero and $H_{11}$ typically decreases with $R e_{d}$, when the porosity is sufficiently large. As seen previously, for a porosity approximately greater or equal to 0.6 the variation of the apparent permeability with the angle $\theta$ is weak in this two-dimensional configuration. For the lowest porosity studied (left frame) the permeability has very small values and the isolines display an irregular behaviour; this is a feature common to all plots relative to the smaller value of $\varepsilon$, signaling that it is probably necessary, in this specific case, to insert additional sample points in building the response surfaces.

In Fig. 14 the parameter $\theta$ is set to $0^{\circ}$ and the response surface is displayed in the $R e_{d}-\phi$ plane. As already indicated, the results confirm that an increase of the Reynolds number is generally associated to a decrease of the first diagonal component of the appar- 

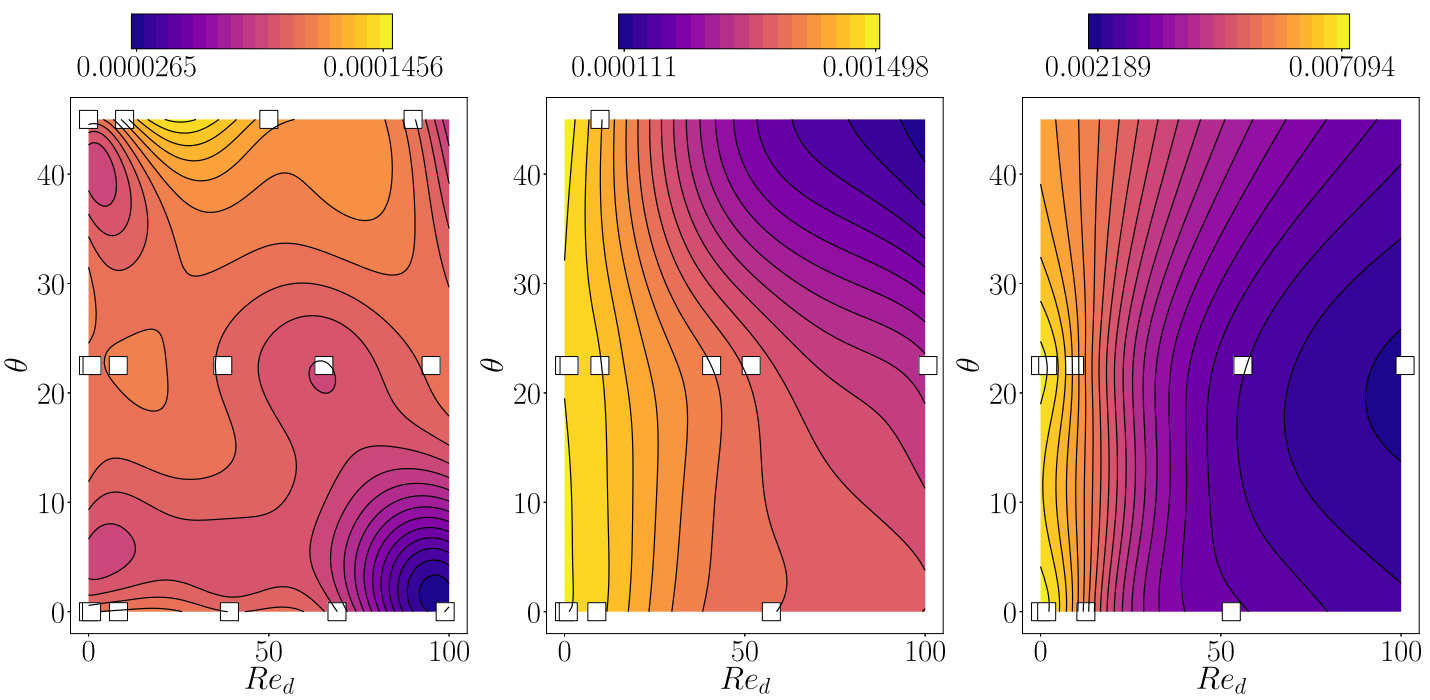

Fig. 13. Response surfaces of $H_{11}$ with $\phi=0^{\circ}$ for porosity $\varepsilon=0.4,0.6,0.8$, from left to right.
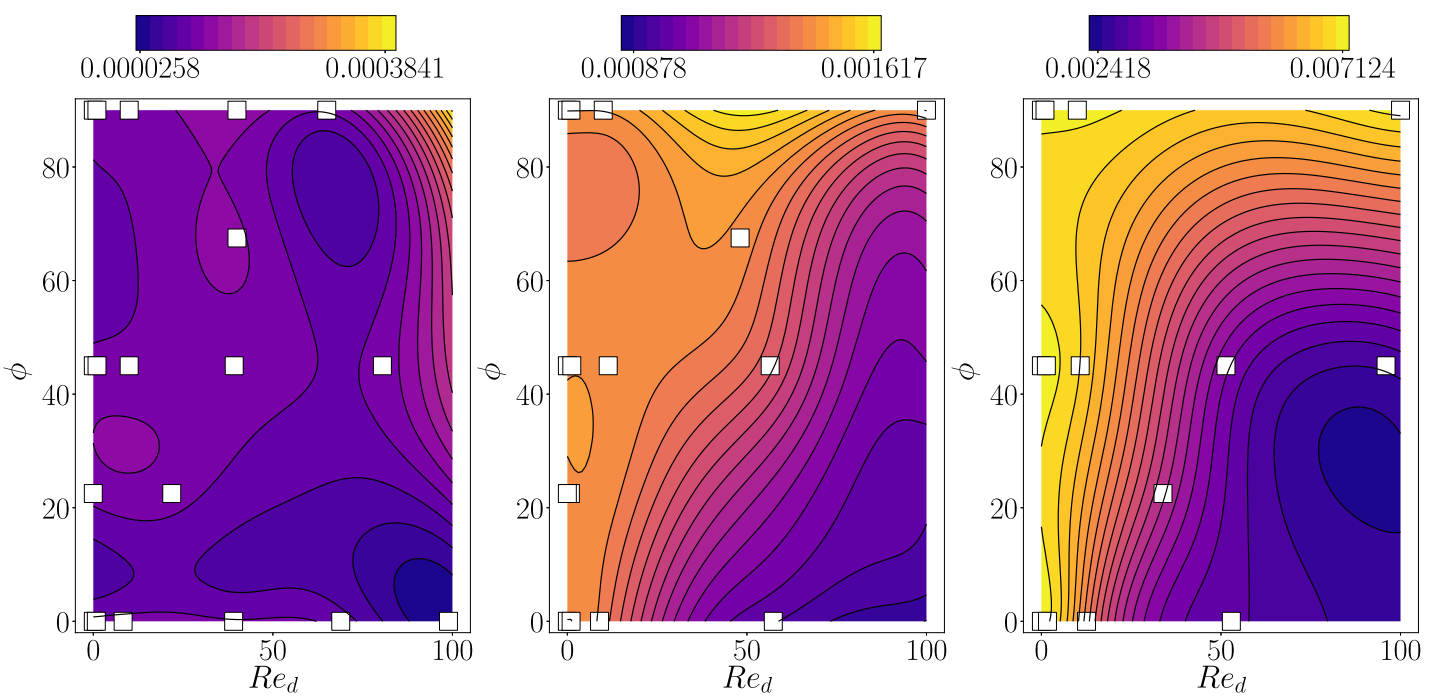

Fig. 14. Response surfaces of $H_{11}$ with $\theta=0^{\circ}$ for porosity $\varepsilon=0.4,0.6,0.8$, from left to right.
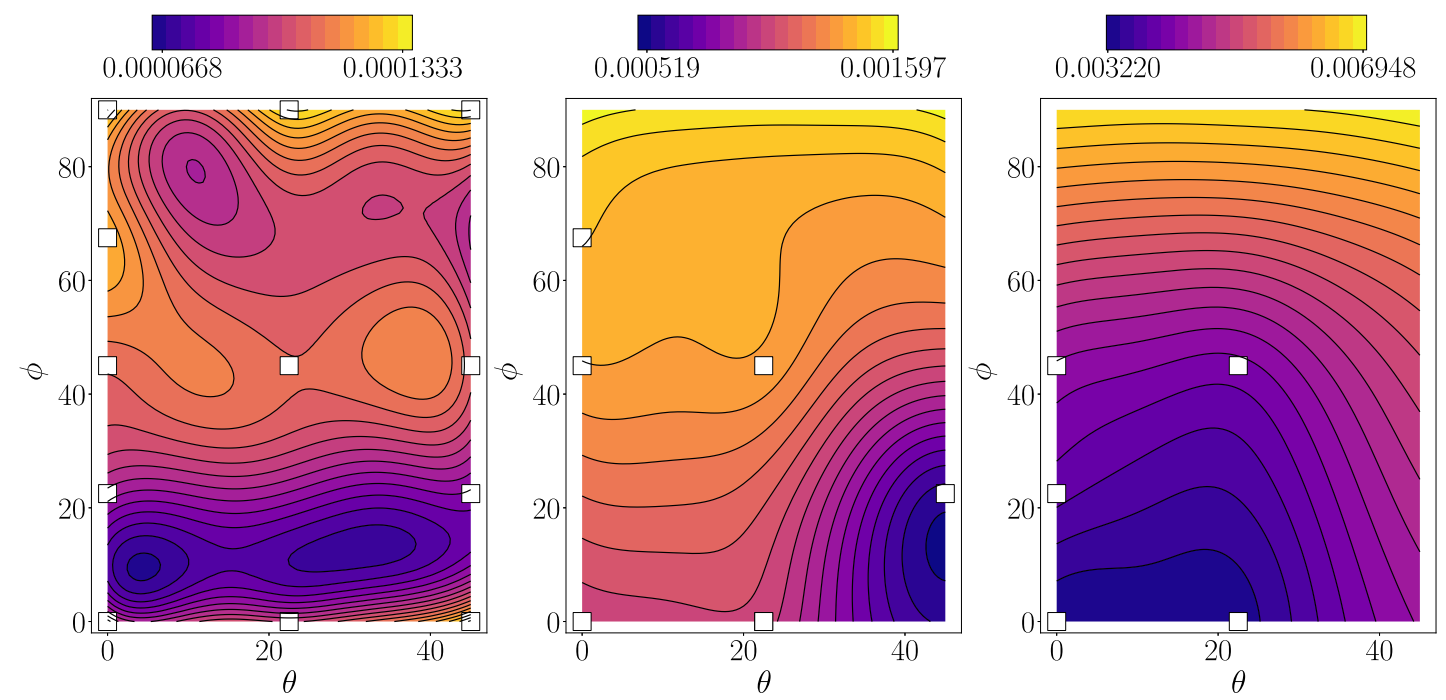

Fig. 15. Response surfaces of $H_{11}$ with $R e_{d}=40$ for porosity $\varepsilon=0.4,0.6,0.8$, from left to right. 

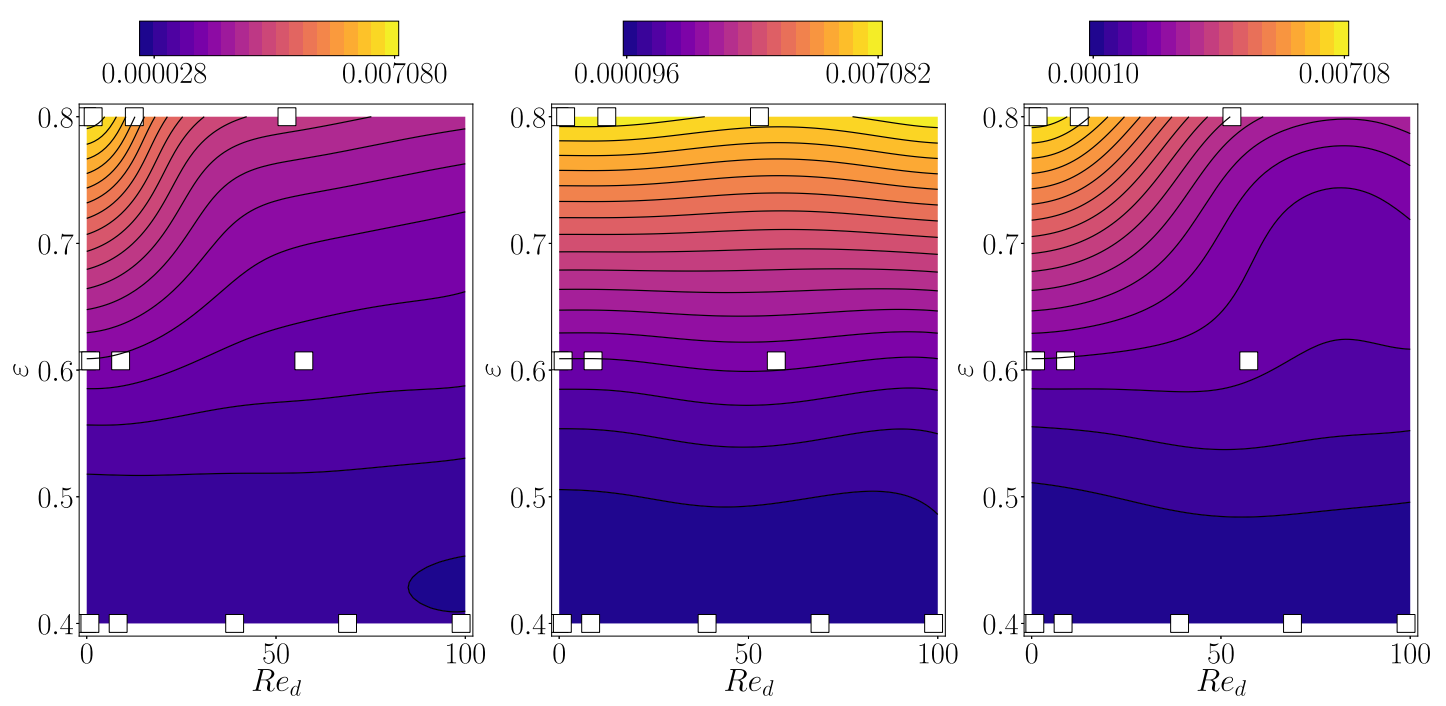

Fig. 16. Response surface of $H_{11}$; in the left frame $\phi=\theta=0^{\circ}$, in the centre frame $\phi=90^{\circ}, \theta=0^{\circ}$ and on the right $\phi=45^{\circ}, \theta=22.5^{\circ}$.

ent permeability tensor. However, the $H_{11}$ variations with respect to $\phi$ are more pronounced than those found with respect to $\theta$ and are due to a real three-dimensionalization of the flow. This conclusion remains to be verified in the lower porosity case (left frame) where the variations are very tiny and more irregular.

In Fig. 15 the Reynolds number is set to the inertial range value of 40 and the response surface is displayed in the $\theta-\phi$ plane. For the two highest porosity values, 0.6 and 0.8 , the results confirm that $H_{11}$ has a much stronger dependence on $\phi$ than on $\theta$, suggesting that the real test of permeability models must include three-dimensional effects. As seen earlier, the behaviour of the permeability when the porosity is low (left frame in the figure) is not intuitive, with a significant effect of the angle $\phi$ and a minor influence of $\theta$. Again this occurs from the constraint provided to the flow by the inclusions, and from the occurrence of a large deviation $\gamma$ in these cases (see Appendix B for the definition of the deviation angle $\gamma$ ).

The response surface is shown in the $R e_{d}-\varepsilon$ plane of Fig. 16 for three sets of $\theta-\phi$ angles. Here a significant effect of the porosity with respect to the Reynolds number is observable. In fact the surface gradient is almost aligned with the porosity direction, i.e. a quasi-Reynolds independence is demonstrated in this plane, and the apparent permeability can change by one order of magnitude in the range of the analysed porosity. Some relatively small Reynolds number effects are visible for $\varepsilon$ equal to 0.8 , when the wake of the flow has more space to develop in the inertial regime. In the central figure the flow is aligned with the direction of the fibers and, as expected, it shows practically no dependence with respect to the Reynolds number.

The response surface analysis has confirmed the qualitative trends which had been reached earlier on the basis of a few selected flow cases, yielding at the same time much more detailed information on the behavior of the apparent permeability with the parameters of the problem. The data base which has been built will be used in future work which will focus, via the VANS approach, on configurations for which neither the porosity nor the local Reynolds number are constant in space or time.

\section{Concluding remarks}

The components of the permeability tensor are essential ingredients for any solution of flow through anisotropic porous media. When the flow through the pores resents of significant acceleration effects, the permeability must be modified (it is then called appar- ent) by the presence of a second tensor, the Forchheimer tensor $\mathbf{F}$, defined by

$\mathbf{F}=\mathbf{K} \mathbf{H}^{-1}-\mathbf{I}$.

The permeability, $\mathbf{K}$, and the apparent permeability, $\mathbf{H}$, can be formally deduced by two closure problems which have been briefly recalled in Section 2. The real obstacle to the solution of the problem for $\mathbf{H}$ is the need to know the microscopic velocity fields through the pores. We have solved for such fields in a unit cell (the REV), varying the forcing amplitude and direction, treating over one hundred different cases of flows through arrangements of parallel fibers. From this, we have thus been able to solve the linear system (10) for all the unknown elements of the intermediate tensor $\mathbf{M}$, from which, through averaging, we obtain the apparent permeability. Such a tensor is indispensable to evaluate accurately the drag force caused by the presence of the fibers, for a macroscopic solution of the flow on the basis of Eqs. (4) and (5) when inertial effects are present.

It has been found that the apparent permeability tensor is strongly diagonally dominant for whatever forcing direction and porosity, provided the microscopic Reynolds number remains below a value approximately equal to 100 ; this results - which is a direct consequence of the transverse isotropy of the material which has been considered here - can be used to compute $\mathbf{H}$ rapidly, approximating it as a diagonal tensor.

Finally, a metamodel has been used to produce results so as to cover the whole space of parameters, and this has allowed the construction of a complete data base. The elements of the data base provide a robust approximation to the "true" apparent permeability values as discussed in Appendix B (see in particular B.2 and B.3). This data base is now being used in simulations of poroelastic media based on the VANS approach.

\section{Acknowledgment}

The authors acknowledge the IDEX Foundation of the University of Toulouse for the financial support granted to the last author under the project Attractivity Chairs. The computations have been conducted at the CALMIP center, Grant no. P1540. The complete database is available at the link: https://github.com/appanacca/ porous_solvers_OF.git. 


\section{Appendix A. Mesh convergence analysis}

The mesh has been computed using the internal OpenFOAM mesher named snappyHexMesh. The final grid is mainly composed by hexahedral cells with a refined regular grid in the boundary layer regions next to the solid surfaces. Three different mesh sizes, with $0.65 \times 10^{6}, 10^{6}$ and $1.5 \times 10^{6}$ elements, have been tested in order to demonstrate spatial convergence. This has been assessed using the Grid Convergence Index (GCI) introduced by Roache (1998).

Images of the coarsest mesh used are shown in Fig. A.17. On the right frame a close up of the grid in the neighbourhood of the fiber's boundary is displayed: twenty points are used in the structured portion of the mesh along the wall-normal direction.

The GCI method is based upon a grid refinement error estimator derived from the theory of generalized Richardson extrapolation. It measures the ratio between the computed value of a quantity over the asymptotic numerical value, thus indicating how far the solution is from the asymptotic ("exact") value. The procedure is simple and provides a method to estimate the order of the spatial convergence, based on two or three different grid sizes. First of all, the grids must be generated with the same algorithm and they must have the same final quality. In each simulation the intrinsic average velocity in the porous medium is sampled. The method follows the following four steps:

1. Estimate the order of convergence of the procedure, defined as $p=\ln \left(\frac{f_{3}-f_{2}}{f_{2}-f_{1}}\right) / \ln r$,

where $r$ is the grid refinement ratio between each grid (it is computed as the ratio between the number of elements of two consecutive grids; the approach imposes that $r$ should remain constant between any couple of consecutive grids and be larger than 1.1 ), and $f_{i}$ represents the amplitude of the intrinsic average velocity in each grid $(1=$ coarse, $2=$ medium and $3=$ fine).

2. Compute the relative error between grid $i$ and $j:|\epsilon|_{i j}=\frac{f_{j}-f_{i}}{f_{i}}$, for $(i, j) \in\{(1,2),(2,3)\}$.

3. Compute $G C I_{i j}=\frac{F_{S}|\epsilon|_{i j}}{r^{p}-1}$, with $F_{S}$ a safety factor equal to 1.25 if the grids are three, and equal to 3 if the grids are only two (Roache, 1998).

4. Check whether each grid level yields a solution that is in the asymptotic range of convergence; this means that the quotient $A C=\frac{G C I_{23}}{G C I_{12}} \frac{1}{r^{p}}$ should be as close as possible to one.
Table A.3

Convergence analysis. Left: average velocity within the REV normalized with $\frac{K_{11}}{v_{\beta}}\|\mathbf{f}\|$, with $K_{11}$ the first component of the tensor K. Right: grid convergence metrics. The REV has $\varepsilon=0.6$, the motion is along $x_{1}$, i.e. $\theta=\phi=0$ and $R e_{d}=180$.

\begin{tabular}{lllll}
\hline $\begin{array}{l}\text { Mesh } \\
\text { index }\end{array}$ & $\begin{array}{l}\text { Mesh } \\
\text { identifier }\end{array}$ & $\begin{array}{l}\text { Average REV } \\
\text { velocity }\end{array}$ & Metric & Value \\
\hline 3 & fine & 1.11 & $\mathrm{GCI}_{23}$ & $0.366 \%$ \\
2 & medium & 1.07 & $\mathrm{GCI}_{12}$ & $1.11 \%$ \\
1 & coarse & 1.09 & $\mathrm{AC}$ & 1.006 \\
\hline
\end{tabular}

The results are summarized in Table A.3.

From the table it can be seen that the intrinsic velocity difference is very small from one grid to the next and the coarse grid provides results close to the expected asymptotic value. This is taken as a sufficiently convincing argument to carry out all the computations in the paper with a grid density equal to that of grid 1 .

\section{Appendix B. Building the metamodel}

\section{B1. DACE sampling}

The first step to build a metamodel is the collection of relevant samples. The quality of the final metamodel strongly depends on the samples collected and their number and distribution is of primary importance.

The apparent permeability tensor, $\mathbf{H}$, depends on four independent variables; the samples have been generated starting from the set of parameters given in Table B.4.

One of the best options to generate the relevant database would be to use a full factorial design approach in which all the combinations of the four variables from Table B.4 are computed. Because of the large number of computations required, this approach has not been retained. We have resorted to the methodology known as DACE (Design and Analysis of Computer Experiments), a technique to fill in the best possible way the space of the parameters of the problem. The Dakota library (Adams et al., 2014) has been selected for the purpose and the Monte-Carlo incremental random sampling algorithm (Giunta et al., 2003) has been chosen, in order to make efficient use of the cases already computed. This incremental approach selects in a quasi-random way the new samples to generate, starting from the existing ones. In the end, the set of samples comprises 118 cases.

In the scatter plot of Fig. B.18 the three diagonal components of the permeability tensor are shown as function of one another. The three porosities are separately considered in each of the above
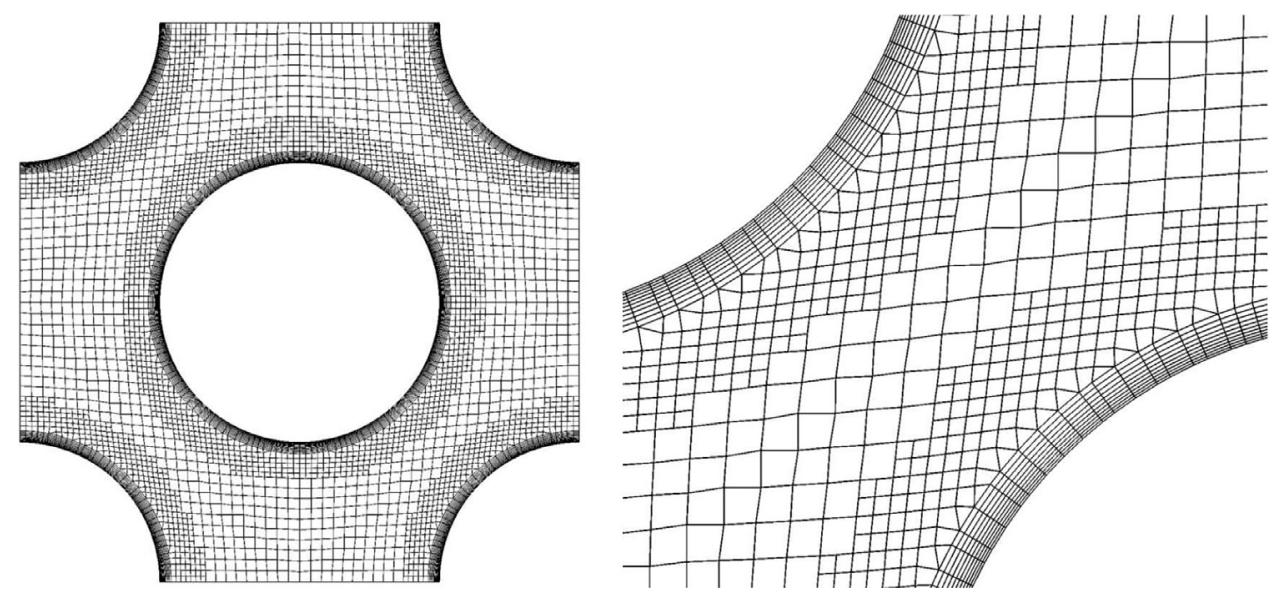

Fig. A.17. Mesh used for the computation; top view (left) and zoom in the boundary layer region (right). $\varepsilon=0.6$. 
Table B.4

Sampling parameters.

\begin{tabular}{llllll}
\hline parameter & values & & & & \\
\hline$\theta$ & $0^{\circ}$ & $22.5^{\circ}$ & $45^{\circ}$ & & \\
$\phi$ & $0^{\circ}$ & $22.5^{\circ}$ & $45^{\circ}$ & $67.5^{\circ}$ & $90^{\circ}$ \\
$\operatorname{Re}_{d}$ & 0 & 10 & 50 & 100 & \\
$\varepsilon$ & 0.4 & 0.6 & 0.8 & & \\
\hline
\end{tabular}

plot, and the permeability points are represented with their linear regression on top. This kind of plot is common in statistical analysis to determine if correlations in the data are present. The permeability components display a positive correlation, with the data points which lie reasonably close to a straight line.

This result has a physical implication. Remembering the diagonal dominance of the permeability tensor, we have in the low $R e_{d}$ limit:

$\left(\left\langle u_{\beta}\right\rangle^{\beta},\left\langle v_{\beta}\right\rangle^{\beta},\left\langle w_{\beta}\right\rangle^{\beta}\right) \sim\left(H_{11} \frac{\partial p}{\partial x_{1}}, H_{22} \frac{\partial p}{\partial x_{2}}, H_{33} \frac{\partial p}{\partial x_{3}}\right)$.

It is then possible to compute the angle between the forcing term, $\nabla p$, and the average velocity vector, represented in Fig. B.19 for the two-dimensional case, $\phi=0$. This is achieved by taking the ratio between the first two components of Darcy's equation, calling $\gamma$ the angular flow deviation with respect to the mean forcing. We thus have:

$\tan (\theta+\gamma)=\frac{H_{22}}{H_{11}} \tan \theta$.

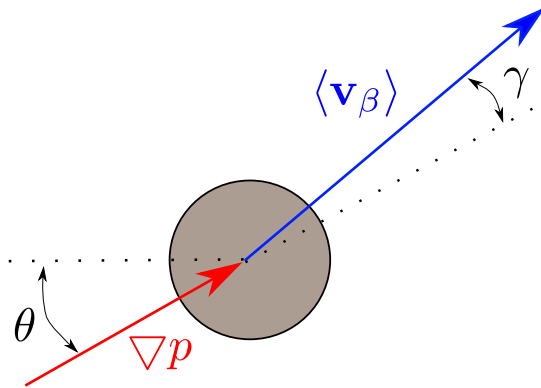

Fig. B.19. Explanatory sketch for the relation between mean pressure gradient and mean velocity field.

If the ratio between the two permeability components is equal to one, the angle $\gamma$ vanishes. The correlation between $H_{11}$ and $H_{22}$ controls the deviation of the flow in the $\left(x_{1}, x_{2}\right)$ plane, and the argument can easily be extended to $H_{11} / H_{33}$ and $H_{22} / H_{33}$ for deviation angles in three-dimensions.

Using a linear correlation such as that shown in Table B.5 and Fig. B.18, it is observed that in the low porosity case $(\varepsilon=0.4)$ the ratio can become very large indicating a strong deviation of the flow from the forcing direction, because of the strong constraint provided by the inclusions. As the porosity increases, the ratio does not differ much from unity, which means that the deviation remains limited. It is simple to see that the deviation angle, for ex-
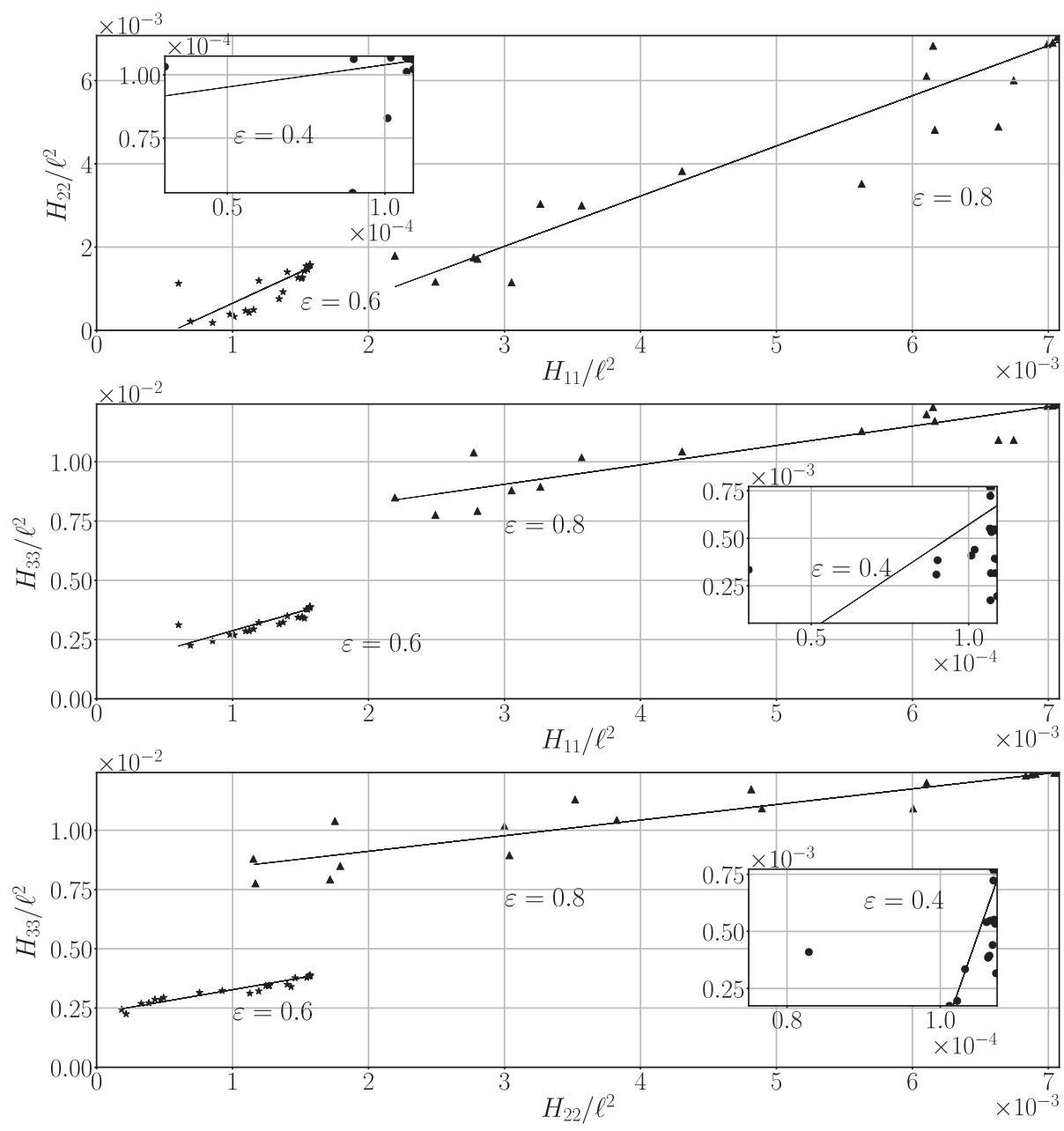

Fig. B.18. Scatter matrix plot for the collected numerical data of the apparent permeability tensor. 
Table B.5

Permeability components ratio for three values of the porosity. The permeability ratios here are given by the angular coefficients of the linear correlations displayed in Fig. B.18.

\begin{tabular}{llll}
\hline$\varepsilon$ & $H_{11} / H_{22}$ & $H_{11} / H_{33}$ & $H_{22} / H_{33}$ \\
\hline 0.4 & 1.57 & 11.06 & 96.03 \\
0.6 & 1.50 & 1.62 & 0.99 \\
0.8 & 1.20 & 0.82 & 0.66 \\
\hline
\end{tabular}

ample in the $\left(x_{1}, x_{2}\right)$ plane, satisfies the approximate relation

$\tan \gamma=\frac{\left(1-\frac{H_{11}}{H_{22}}\right) \tan \theta}{\frac{H_{11}}{H_{22}}+\tan ^{2} \theta}$,

so that for $\frac{H_{11}}{H_{22}}$ equal to, say, 1.5, the largest deviation remains always below $12^{\circ}$ for any $\theta$. It should however be kept in mind that trends based on these ratios are valid only as long as Darcy's law and linear correlations are acceptable. Cases exists for which such trends are violated; for example, a flow with $\theta=45^{\circ}$ and $\phi=0^{\circ}$ has deviation angle $\gamma$ equal to zero, for whatever porosity. In this case $H_{11} / H_{22}$ is equal to one and such a point is an outlier in the regression plots of Fig. B.18.

\section{B2. Kriging interpolation method}

The kriging approach is a linear interpolation/extrapolation method that aims to build a predictor field based on a set of observations $\left(\mathbf{x}_{\mathbf{i}}, \mathbf{y}\left(\mathbf{x}_{\mathbf{i}}\right)\right)$, for $i=1, \ldots, n$.

The predictor $\hat{f}(\mathbf{x})$ is a sum of a trend function $t(\mathbf{x})$ and a Gaussian process error model $e(\mathbf{x})$ :

$\hat{f}(\mathbf{x})=t(\mathbf{x})+e(\mathbf{x})$.

The aim of the error model is to make adjustments on the trend function so that, for any point of the sampling the predictor is exactly equal to the sample, i.e. $\hat{f}\left(\mathbf{x}_{\mathbf{i}}\right)=y\left(\mathbf{x}_{\mathbf{i}}\right)$. This property represents one of the main qualities of this approach. In addition, when the model parameters are conveniently set, the trend function and the covariance model can take into account both smooth and steep variations in the data set.

The trend function defined here is based on a second order least-square regression, with the coefficients found from the solution of the associated linear system. The Gaussian process error model has zero-mean and its covariance between two generic data-points, $x_{i}$ and $x_{j}$, is written as

$\operatorname{Cov}\left(y\left(\mathbf{x}_{\mathbf{i}}\right), y\left(\mathbf{x}_{\mathbf{j}}\right)\right)=\sigma^{2} r\left(\mathbf{x}_{\mathbf{i}}, \mathbf{x}_{\mathbf{j}}\right)$.

The coefficient $\sigma$ is an amplitude parameter and $r\left(x^{i}, x^{j}\right)$ is a correlation function, based on the Matérn covariance model, that reads:

$r\left(\mathbf{x}_{\mathbf{i}}, \mathbf{x}_{\mathbf{j}}\right)=\frac{2^{1-v}}{\Gamma(v)}\left(\frac{\sqrt{2} v\left|\mathbf{x}_{\mathbf{i}}-\mathbf{x}_{\mathbf{j}}\right|}{|\lambda|}\right)^{v} K_{v}\left(\frac{\sqrt{2} v\left|\mathbf{x}_{\mathbf{i}}-\mathbf{x}_{\mathbf{j}}\right|}{|\lambda|}\right)$,

where $K_{v}($.$) is a modified Bessel function and \Gamma($.$) is the gamma$ function. The parameters that can be used to tune the metamodel are the amplitude parameter $\sigma$, the exponent $v$ and the scale vector $\lambda$. The kriging metamodel outputs can show different behaviours for different selections of the above three parameters and their setting is thus crucial. The amplitude parameter $\sigma$ is chosen to be equal to 1 ; larger value lead to steeper gradients and undesirable local extrema around the data points. The vector $\lambda=\left(\lambda_{\theta}, \lambda_{\phi}, \lambda_{R_{d}}, \lambda_{\varepsilon}\right)$ is a scaling parameter for the distance $\left|\mathbf{x}_{\mathbf{i}}-\mathbf{x}_{\mathbf{j}}\right|$. In this study, through systematic variations of the

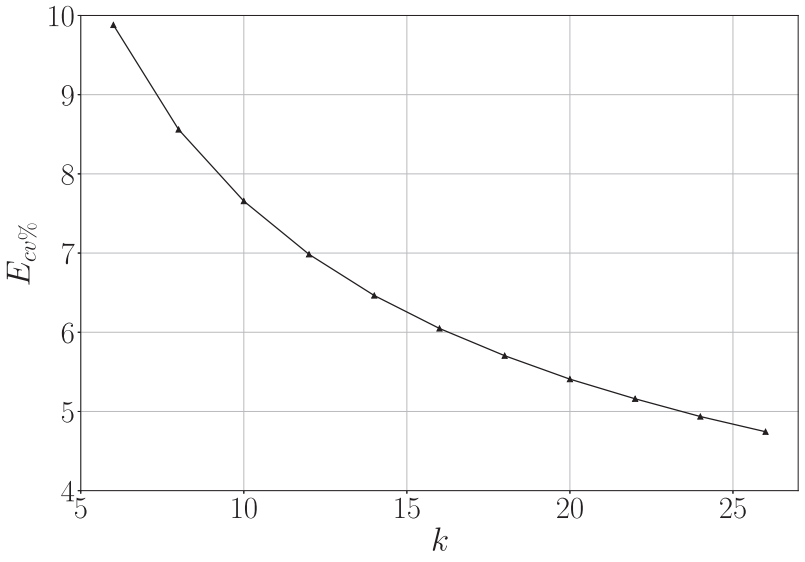

Fig. B.20. Relative mean error computed using the $k$-fold approach presented against the number of folds $k$ used to divide the dataset.

parameters it is found that the choice $\lambda=(1.2,1,1,1)$ yields acceptable results; in particular, the weight along $\theta$ is mildly larger than in the other directions in order to obtain smoother metamodel surfaces in this direction. The exponent $v$ controls the covariance function and more particularly its gradients. When $v=$ $1 / 2$ the covariance can be approximated by a negative exponential, $\exp (-\alpha x)$ and when $v$ goes to infinity it behaves as $\exp \left(-\alpha x^{2}\right)$. In the present study, the best (i.e. smoother) results are obtained for $v$ equal to 1.9. The above parameters have been chosen in order to avoid unphysical or unrealistic behavior of the apparent permeability such as, for instance, negative values or steep, spurious local maxima/minima. The method above is implemented in OpenTURNS (Baudin et al., 2016).

\section{B3. Robustness of the metamodel}

A procedure called $k$-fold, belonging to the class of crossvalidation methods, has been used in order to prove the robustness of the metamodel. The $k$-fold method starts with the full database $S_{n}=\left(\mathbf{x}_{\mathbf{i}}, \mathbf{y}\left(\mathbf{x}_{\mathbf{i}}\right)\right)$, for $i=1, \ldots, n$, split into two complementary sets of size $n_{1}$ and $n_{2}$, such that $S_{n}=S_{n_{1}} \cup S_{n_{2}}$. Then, a new metamodel is built using only the points present in the set $S_{n_{1}}$. For the sake of clarity, the metamodel built with only the subset $S_{n_{1}}$ will be called from now on $\hat{f}^{n_{1}}$, and the metamodel built with all the database will be indicated as $\hat{f}^{n}$. The idea now is to use the points in the set $S_{n_{2}}$ as test, since they are essentially "new" for the metamodel $\hat{f}^{n_{1}}$. The division of the subset is performed picking points in a random way, and is repeated $k$ times in order to rule out any possible "lucky" combination.

Thus, the metric used for the error computation is the following:

$\xi_{c v}=\frac{1}{k n_{2}} \sum_{i=1}^{k} \sum_{j=1}^{n_{2}}\left(\hat{f}_{i}^{n}\left(x_{j}\right)-\hat{f}_{i}^{n_{2}}\left(x_{j}\right)\right)^{2}$,

quantifying the quadratic error between the original metamodel and the one built each time with a different set that belongs to different folds.

The metric is also averaged over all the test points $n_{2}$ present in all the $k$ folds. The relative mean error can be computed as:

$E_{c v \%}=100 \frac{\sqrt{\xi_{c v}}}{\operatorname{mean}\left(\left|\hat{f}_{i}^{n}\right|\right)}$.

In our case the number of points used to test the model $n_{2}$ is equal to $\sqrt{N} \approx 12$ as recommended for kriging metamodels by Wang and Shan (2007). The number of folds has been varied from 5 to 25 
and in all the cases tested the $E_{c v \%}$ has been found to decrease below $6 \%$ when we use at least 16 folds (which means leaving out 7 to 8 points from the metamodel construction), which is more than acceptable to prove that our kriging method is a robust approximation (Fig. B.20).

\section{References}

Adams, B.M., Bauman, L.E., B/ohnhoff, W.J., Dalbey, K.R., Ebeida, M.S., Eddy, J.P., Eldred, M.S., Hough, P.D., Hu, K.T., Jakeman, J.D., Stephens, J.A., Swiler, L.P., Vigil, D.M., Wildey, T.M., 2014. Dakota, A Multilevel Parallel Object-Oriented Framework for Design Optimization, Parameter Estimation, Uncertainty Quantification, and Sensitivity Analysis: Version 6.0 Theory Manual. Technical Report. Sandia National Laboratories, SAND2014-4253.

Agnaou, M., Lasseux, D., Ahmadi, A., 2016. From steady to unsteady laminar flow in model porous structures: an investigation of the first Hopf bifurcation. Comput. Fluids 136, 67-82.

Baudin, M., Dutfoy, A., Iooss, B., Popelin, A.L., 2016. OpenTURNS: An Industrial Software for Uncertainty Quantification in Simulation. Springer International Publishing.

Breugem, W.P., 2005. The influence of wall permeability on laminar and turbulent flows. Theory and simulations. Delft University of Technology, The Netherlands.

Brinkman, H.C., 1949. A calculation of the viscous force exerted by a flowing fluid on a dense swarm of particles. Appl. Sci. Res. 1 (1), 27-34.

Carman, P.C., 1937. Fluid flow through granular beds. Trans. Inst. Chem. Eng. 15, $150-166$.

Darcy, H., 1856. Les fontaines publiques de la ville de Dijon. Victor Dalmont.

Edwards, D.A., Shapiro, M., Bar-Yoseph, P., Shapira, M., 1990. The influence of Reynolds number upon the apparent permeability of spatially periodic arrays of cylinders. Phys. Fluids 2 (1), 45-55.

Ergun, S., Orning, A.A., 1949. Fluid flow through randomly packed columns and fluidized beds. Ind. Eng. Chem. 41 (6), 1179-1184.
Forchheimer, P.H., 1901. Wasserbewegung durch boden. Zeitz. Ver. Duetch Ing. 45, 1782-1788.

Giunta, A.A., Wojtkiewicz, S.F., Eldred, M.S., 2003. Overview of modern design of experiments methods for computational simulations. In: Proceedings of the 41st AIAA Aerospace Sciences Meeting and Exhibit, 6-9 January 2003, Reno, Nevada. AIAA-2003-0649.

Kleijnen, J.P.C., 2017. Regression and kriging metamodels with their experimental designs in simulation: a review. Eur. J. Oper. Res. 256 (1), 1-16.

Kozeny, J., 1927. Über grundwasserbewegung. Wasserkraft und Wasserwirtschaft 22 (5), 67-70.

Lasseux, D., Arani Abbasian, A., Ahmadi, A., 2011. On the stationary macroscopic inertial effects for one phase flow in ordered and disordered porous media. Phys. Fluids 23 (7), 073103

Pauthenet, M., Davit, Y., Quintard, M., Bottaro, A., 2017. Topological scaling for inertial transition in porous media. Submitted.

Roache, P.J., 1998. Verification and Validation in Computational Science and Engineering. Hermosa Press, Albuquerque, NM.

Soulaine, C., Quintard, M., 2014. On the use of a Darcy-Forchheimer like model for a macro-scale description of turbulence in porous media and its application to structured packings. Int J Heat Mass Transf. 74, 88-100.

Wang, G.G., Shan, S., 2007. Review of metamodeling techniques in support of engineering design optimization. J. Mech. Des. 129 (4), 370-380.

Weller, H.G., Tabor, G., Jasak, H., Fureby, C., 1998. A tensorial approach to computational continuum mechanics using object-oriented techniques. Comput. Phys. 12 (6), 620-631.

Whitaker, S., 1986. Flow in porous media i: a theoretical derivation of Darcy's law. Transp. Porous Media 1 (1), 3-25.

Whitaker, S., 1996. The Forchheimer equation: a theoretical development. Transp. Porous Media 25 (1), 27-61.

Whitaker, S., 2013. The Method of Volume Averaging. Springer Science \& Business Media.

Yazdchi, K., Srivastava, S., Luding, S., 2011. Microstructural effects on the permeability of periodic fibrous porous media. Int. J. Multiphase Flow 37 (8), 956-966.

Zampogna, G.A., Bottaro, A., 2016. Fluid flow over and through a regular bundle of rigid fibres. J Fluid Mech. 792, 5-35. 